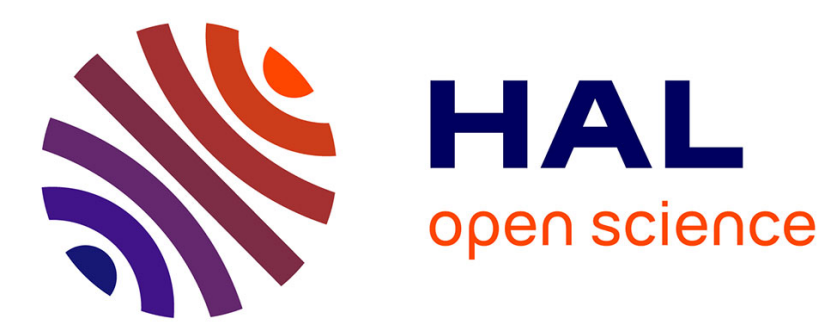

\title{
Time evolution of discrete fourth-order elliptic operators
}

\author{
M Ben-Artzi, J.-P Croisille, D Fishelov
}

\section{To cite this version:}

M Ben-Artzi, J.-P Croisille, D Fishelov. Time evolution of discrete fourth-order elliptic operators. Numerical Methods for Partial Differential Equations, 2019, 10.1002/num.22358 hal-03264826

\section{HAL Id: hal-03264826 \\ https://hal.science/hal-03264826}

Submitted on 18 Jun 2021

HAL is a multi-disciplinary open access archive for the deposit and dissemination of scientific research documents, whether they are published or not. The documents may come from teaching and research institutions in France or abroad, or from public or private research centers.
L'archive ouverte pluridisciplinaire HAL, est destinée au dépôt et à la diffusion de documents scientifiques de niveau recherche, publiés ou non, émanant des établissements d'enseignement et de recherche français ou étrangers, des laboratoires publics ou privés. 


\title{
TIME EVOLUTION OF DISCRETE FOURTH-ORDER ELLIPTIC OPERATORS
}

\author{
MATANIA BEN-ARTZI, J.-P. CROISILLE, AND D. FISHELOV
}

AbstraCt. The evolution equation

$$
\frac{\partial}{\partial t} u=-\left(\frac{\partial}{\partial x}\right)^{4} u+A(x)\left(\frac{\partial}{\partial x}\right)^{2} u+A^{\prime}(x)\left(\frac{\partial}{\partial x}\right) u-B(x) u+f, \quad x \in \Omega=[0,1], t \geq 0,
$$

is considered. A discrete parabolic methodology is developed, based on a discrete elliptic (fourth-order) calculus. The main ingredient of this calculus is a discrete biharmonic operator (DBO). In the general case, it is shown that the approximate solutions converge to the continuous one. An "almost optimal" convergence result $\left(O\left(h^{4-\varepsilon}\right)\right)$ is established in the case of constant coefficients, in particular in the pure biharmonic case. Several numerical test cases are presented, that not only corroborate the theoretical accuracy result, but also demonstrate high-order accuracy of the method in nonlinear cases. The nonlinear equations include the well studied Kuramoto-Sivashinsky equation. Numerical solutions for this equation are shown to approximate remarkably well the exact solutions. The numerical examples demonstrate the great improvement achieved by using the DBO instead of the standard (five-point) discrete bilaplacian.

\section{INTRODUCTION}

Let $\Omega=[0,1]$ and consider the linear evolution equation associated with the general one-dimensional fourth-order elliptic operator

$$
\mathcal{L}_{A, B} z(x)=\left(\frac{d}{d x}\right)^{4} z(x)-A(x)\left(\frac{d}{d x}\right)^{2} z(x)-A^{\prime}(x)\left(\frac{d}{d x}\right) z(x)+B(x) z(x)
$$

The associated evolution equation is therefore,

$$
\begin{gathered}
\frac{\partial}{\partial t} u(x, t)=-\mathcal{L}_{A, B} u(x, t)+f(x) \\
=-\left(\frac{\partial}{\partial x}\right)^{4} u(x, t)+A(x)\left(\frac{\partial}{\partial x}\right)^{2} u(x, t)+A^{\prime}(x)\left(\frac{\partial}{\partial x}\right) u(x, t)-B(x) u(x, t)+f(x), \quad x \in \Omega=[0,1], t \geq 0,
\end{gathered}
$$

where we assume that

$$
A(x), B(x), f(x) \text { are real continuous functions, and } A(x) \in C^{1}(\Omega) .
$$

The equation is supplemented by the initial data

$$
u(x, 0)=u_{0}(x)
$$

and homogeneous boundary conditions

$$
u(0, t)=\frac{\partial}{\partial x} u(0, t)=u(1, t)=\frac{\partial}{\partial x} u(1, t)=0, \quad t \geq 0 .
$$

The initial function $u_{0}$ is assumed to be sufficiently smooth, and for simplicity we take $u_{0} \in C^{\infty}(\Omega)$. To justify our interest in this equation, we can gratefully quote from the Introduction of [27]: "In recent years, fourthorder problems have also become important in image processing and for modeling diffusion processes in physics and material sciences." This paper deals with high-order discrete approximations to (1.2), and is a sequel to the "elliptic" paper [8]. It builds on the premise of a discrete functional calculus. The approximation procedure consists of functions and operators that are defined on a fixed grid; every ingredient of the continuous theory (not only functions and operators but also fundamental properties such as coercivity) has its discrete counterpart. There is no reference to any piecewise continuous functions, as would be the case with finite element or discontinuous Galerkin methods. An important feature of the discrete operators is their compactness, namely, depending only

Date: December 10, 2018.

2010 Mathematics Subject Classification. Primary 65M06; Secondary 65N12, 65M15.

Key words and phrases. fourth order parabolic equation, discrete elliptic calculus, discrete evolution, biharmonic operator, Hermitian derivative, optimal convergence, boundary values, Kuramoto-Sivashinsky equation. 
on nearest neighbors. The convergence of the discrete solutions to the exact one is measured solely on the grid, as it is being refined. A complete convergence proof in the general linear case is presented. In the constant coefficient case it is shown to be "almost optimal", as is further discussed below. This discretization methodology enables us to go beyond the linear case and consider the approximation to various well-known nonlinear evolution equations. Since it has already been extensively implemented for the $2-D$ Navier-Stokes system (see further below), we have chosen here to test it on the Kuramoto-Sivashinsky equation. This is carried out in the last section of the paper.

There is vast literature pertaining to the discrete approximation of higher order (in the spatial coordinates) evolution equations. The studies are divided to "categories" reflecting either interest in specific physical application or accounting for a particular numerical method. It would take a very comprehensive survey, beyond the scope of this paper, to give a full account of the literature. We shall just mention six representative examples (all including extensive bibliographies).

- The papers $[9,11,23]$ focus mainly on the issue of an accurate discrete time stepping. The first deals with the biharmonic modified forward time-stepping; the highest order biharmonic operator is treated implicitly. The second deals with extensions of the IMEX approach to high order semi-implicit schemes, mostly to second-order (in space) reaction-diffusion equations. The third introduces the modified exponential timedifferencing (ETD) method; the linear (say, biharmonic) part is eliminated by an "integrating factor", so that the nonlinear term can be separately handled by a suitable Runge-Kutta method.

- In [9] the physical models of interest are the evolution of thin films and phase fields. The underlying assumption is that the fourth-order (spatial) elliptic problem at each step is accurately handled. In particular, periodic boundary conditions are imposed, avoiding the issue of boundary layers. The same type of physical applications is considered in [2], which provides an overview of finite volume and finite element methods, in one or two space dimensions.

- The adaptive mesh methodology in [27] is applied to the Cahn-Hilliard equation modeling phase separation. The spatial part is handled using collocation by (piecewise) seventh-order Hermite polynomial.

- In the area of image processing various models involving fourth-order evolution equations are extensively studied. In [20] a non-linear fourth-order equation, which is used for noise reduction and simplification of two dimensional images, was investigated for the existence and uniqueness of solutions of the problem. In [19] a Navier-Stokes system in streamfunction formulation with a non-linear viscosity term was proposed and tested for image impainting. In [26] fourth-order partial differential equations were used for noise removal of Magnetic Resonance Images.

- In [32, Section 3] a local discontinuous Galerkin (LDG) method is developed for biharmonic type equations, primarily in one space dimension. Their method is based on introducing new unknowns (related to successive derivatives of the solution) and then approximating the resulting first-order system. In [13] this reduction was circumvented by using fluxes (at cell boundaries) associated with higher order derivatives.

- Optimal error analysis was carried out on finite element methods in the following papers. In [14] the author approximates a fourth-order time dependent differential equation by a finite element method and establishes optimal error estimates for the semi-discrete case. In [32, Section 3], an optimal convergence rate for the LDG method mentioned above was achieved. In [21] the authors treat the biharmonic problem using a conformal bicubic Hermite polynomial. Using interpolation theory it may be shown that if the solution is smooth enough then the error is bounded by $C h^{4}$, where $h$ is the mesh size.

It should be emphasized that all treatments of the time dependent problems rely on a careful high order approximation of the biharmonic operator. Recently various LDG methods were proposed. A main tool is the interior penalty procedure, that must be properly chosen [30]. It is beyond the scope of this paper to compare the compact scheme used here for $\partial_{x}^{4}$ with any variant of the DG methodology. We refer to [8] for a further discussion on this topic.

One of the oldest instances of physical models in which the biharmonic operator plays a crucial role is the $2-D$ Navier-Stokes system in pure streamfunction formulation [6]. Indeed, the present study grew out of earlier work on high order approximation of this system [3, 4, 24].

The one-dimensional scheme was already extended to a two dimensional one in [5]. A non-optimal analysis was carried out for a second order compact scheme for the full nonlinear Navier-Stokes system in [4]. It was shown that the convergence rate is at least 3/2. That is suboptimal compared to the actual convergence rate (order 2), that was verified in numerical results. 
In [7] we developed a fast direct solver in two dimensions for the biharmonic problem in a rectangular grid. It is based on FFT expansions and on the Sherman-Morrison formula.

Our convergence analysis here is different from standard Summation by Parts arguments. Instead, it is based on a detailed discrete functional calculus. It runs parallel to the classical PDE theory and allows us to establish in a fully discrete context the coercivity properties of the operators (see Section 3). In turn these properties enable us to prove convergence, not only for the solution but also for its derivatives. In fact, the optimal (4-th order) convergence of the discrete biharmonic operator ( see Claim 4.1 below) was established in [6, Chapter 10] by invoking matrix methods. We refer to [8] for a detailed discussion.

The paper is organized as follows. In Section 2 we recall the basic well-posedness facts related to Equation (1.2) and in Section 3 we review the construction of the discrete (elliptic) operators, the analogs of the second-order and fourth-order derivatives. In particular, the discrete biharmonic operator (DBO) is defined in (3.11). The construction of the discrete operators depends in a fundamental way on the concept of Hermitian derivative, which is defined in (3.9). The present paper aims at proving (in the general case) the convergence of the discrete approximation to the continuous solution and establishing (in the constant coefficient case) the significant (from both theoretical and practical aspects) "high-order" accuracy of the approximation. The crucial point in obtaining such estimates is that the discrete operators possess the fundamental elliptic properties (such as coercivity), in full analogy with the continuous elliptic operators.

In Section 4 we study the pure biharmonic evolution equation, and prove the "almost optimal" convergence rate (see below a more detailed explanation). We treat this case first because of its great importance as a model in many classical problems of mathematical physics, such as elasticity theory or the streamfunction formulation of the $2-D$ Navier-Stokes system.

In Section 5 we introduce (Equation (5.1)) the discrete elliptic operator $\mathcal{L}_{A, B, h}$, which is the discrete analog to $\mathcal{L}_{A, B}$. The semi-discrete equation, analogous to (1.2) is given in (5.2):

$$
\frac{\partial}{\partial t} \mathfrak{v}\left(x_{j}, t\right)=-\mathcal{L}_{A, B, h} \mathfrak{v}\left(x_{j}, t\right)+f^{*}\left(x_{j}\right), \quad 1 \leq j \leq N-1, t \geq 0 .
$$

The unknown function $\mathfrak{v}\left(x_{j}, t\right)$ is a grid function defined on the discrete spatial grid and on the continuous time variable. By the "convergence of the scheme" we mean the convergence (in an appropriate sense) of the functions (in time) $\mathfrak{v}\left(x_{j}, t\right), t \geq 0$ to the corresponding restrictions of the exact solution $u\left(x_{j}, t\right), t \geq 0$ as the number $N$ of the grid points increases. The convergence of the discrete scheme to the exact solution is stated in Theorem 5.3.

In Section 6, the general constant coefficient case $(A(x) \equiv a, B(x) \equiv b)$ is studied. We show that it can be reduced to the pure biharmonic case. To measure the accuracy of the scheme, we estimate the difference between the grid function and the restriction of the exact solution in terms of a power $O\left(h^{\beta}\right)$, where $h=\frac{1}{N}$ is the mesh size (we consider here only uniform meshes). The exponent $\beta>0$ is the "order of accuracy". An estimate with $\beta=4$ is an "optimal" convergence rate. Even though we believe (based on the numerical test cases) that our scheme yields the optimal convergence rate, it remains an open problem (even for the pure biharmonic case). We are able to show (Theorem 6.2) only "almost optimal" convergence rate, meaning that we can take $\beta=4-\varepsilon$ for any $\varepsilon>0$ (but with a coefficient that may blow up as $\varepsilon \rightarrow 0$ ).

In Section 7 we present a collection of numerical examples that demonstrate the convergence properties claimed in the preceding sections. It is worthwhile pointing out the great improvement in accuracy achieved by using the DBO instead of the standard (five-point) bilaplacian. This is explicitly demonstrated in the fourth test case.

In fact, we go beyond the linear theory expounded here and present numerical simulations of several nonlinear evolution equations. In particular, we consider the well-studied Kuramoto-Sivashinsky equation in the seventh test case.

In the Appendix we give details of the Taylor expansions of the finite difference operators addressed in the paper. For these expansions we give the nominal order, namely disregarding boundary conditions.

\section{THE LINEAR EVOLUTION EQUATION-BASIC FACTS}

As is well-known, non-homogeneous boundary conditions are accommodated by a modification of the righthand side function $f(x)$. We use the standard notation for Sobolev spaces; $H^{p}(\Omega)$ is the space of functions with (distributional) derivatives that are square integrable up to order $p$, while $H_{0}^{p}(\Omega)$ is the closure of $C_{0}^{\infty}(0,1)$ in $H^{p}$. 
Defining a function $w(x, t)=e^{c t} u(x, t)$, for some positive constant $c>0$, we see that $w$ satisfies the equation

$$
\frac{\partial}{\partial t} w(x, t)=-\mathcal{L}_{A, B+c} w(x, t) .
$$

Remark that the initial and boundary conditions of $w$ are identical to those of $u$. By taking $c>0$ sufficiently large, and without changing notation, we can therefore assume that $\mathcal{L}_{A, B}$ is coercive in the sense that,

Claim 2.1. [1, Theorem 7.5] There exists a constant $C>0$ such that, for every function $z(x) \in H^{4}(\Omega)$, satisfying the homogeneous boundary conditions

$$
z(0)=z^{\prime}(0)=z(1)=z^{\prime}(1)=0,
$$

we have

$$
\int_{0}^{1} \mathcal{L}_{A, B} z(x) \cdot z(x) d x \geq C \int_{0}^{1}\left[z^{\prime \prime}(x)^{2}+z^{\prime}(x)^{2}+z(x)^{2}\right] d x .
$$

By a standard argument about parabolic equations it follows that Equation (1.2) has a unique solution $u(x, t)$.

Assume that the initial function $u_{0} \in C^{\infty}(\Omega)$. Note that in view of the homogeneous boundary conditions (1.4) all time derivatives of the solution vanish on the boundary. By differentiating the equation $N \geq 1$ times with respect to $t$, and taking the scalar product with $\left(\frac{\partial}{\partial t}\right)^{N} u(x, t)$ we obtain

$$
\begin{aligned}
& \frac{1}{2} \frac{d}{d t} \int_{0}^{1}\left|\left(\frac{\partial}{\partial t}\right)^{N} u(x, t)\right|^{2} d x=-\int_{0}^{1}\left|\left(\frac{\partial}{\partial x}\right)^{2}\left(\frac{\partial}{\partial t}\right)^{N} u(x, t)\right|^{2} d x \\
& +\int_{0}^{1} A(x)\left|\frac{\partial}{\partial x}\left(\frac{\partial}{\partial t}\right)^{N} u(x, t)\right|^{2} d x-\int_{0}^{1} B(x)\left|\left(\frac{\partial}{\partial t}\right)^{N} u(x, t)\right|^{2} d x .
\end{aligned}
$$

The integrations-by-parts in the right-hand side are justified by the boundary conditions (1.4). Invoking the Sobolev inequality (with $\phi=\left(\frac{\partial}{\partial t}\right)^{N} u(x, t)$ )

$$
\int_{0}^{1}\left|\phi^{\prime}(x)\right|^{2} d x \leq \varepsilon \int_{0}^{1}\left|\phi^{\prime \prime}(x)\right|^{2} d x+C(\varepsilon) \int_{0}^{1}|\phi(x)|^{2} d x,
$$

and taking a sufficiently small $\varepsilon>0$ we infer that

$$
\frac{d}{d t} \int_{0}^{1}\left|\left(\frac{\partial}{\partial t}\right)^{N} u(x, t)\right|^{2} d x \leq C \int_{0}^{1}\left|\left(\frac{\partial}{\partial t}\right)^{N} u(x, t)\right|^{2} d x,
$$

where $C>0$ depends only on $A(x), B(x)$. Gronwall's lemma now yields

$$
\int_{0}^{1}\left|\left(\frac{\partial}{\partial t}\right)^{N} u(x, t)\right|^{2} d x \leq e^{C t} \int_{0}^{1}\left|\left(\frac{\partial}{\partial x}\right)^{4 N} u_{0}\right|^{2} d x, \quad t \geq 0 .
$$

The equation (1.2) enables us to replace temporal derivatives by spatial derivatives, to any order, so the Sobolev embedding theorem yields, with a constant $C\left(N, T, u_{0}\right)>0$, depending only on $u_{0}, N, T$,

$$
\sup _{x \in[0,1]}\left|\left(\frac{\partial}{\partial t}\right)^{N} u(x, t)\right| \leq C\left(N, T, u_{0}\right), \quad t \in[0, T] .
$$




\section{THE BASIC SETUP for DISCRETE APPROXIMATION}

We equip the interval $\Omega=[0,1]$ with a uniform grid

$$
x_{j}=j h, \quad 0 \leq j \leq N, \quad h=\frac{1}{N} .
$$

The approximation is carried out by grid functions $\mathfrak{v}$ defined on $\left\{x_{j}, 0 \leq j \leq N\right\}$. The space of these grid functions is denoted by $l_{h}^{2}$. For their components we use either $\mathfrak{v}_{j}$ or $\mathfrak{v}\left(x_{j}\right)$. For every smooth function $G(x)$ we define its associated grid function

$$
G_{j}^{*}=G\left(x_{j}\right), \quad 0 \leq j \leq N .
$$

Clearly $G^{*}$ depends on the parameter $h=\frac{1}{N}$, but we do not indicate this dependence in order to simplify the notation. The discrete $l_{h}^{2}$ scalar product is defined by

$$
(\mathfrak{v}, \mathfrak{w})_{h}=h \sum_{j=0}^{N} \mathfrak{v}_{j} \mathfrak{w}_{j},
$$

and the corresponding norm is

$$
|\mathfrak{v}|_{h}^{2}=h \sum_{j=0}^{N} \mathfrak{v}_{j}^{2} .
$$

For linear operators $\mathcal{A}: l_{h}^{2} \rightarrow l_{h}^{2}$ we use $|\mathcal{A}|_{h}$ to denote the operator norm. The discrete sup-norm is

$$
|\mathfrak{v}|_{\infty}=\max _{0 \leq j \leq N}\left\{\left|\mathfrak{v}_{j}\right|\right\} .
$$

The discrete homogeneous space of grid functions is defined by

$$
l_{h, 0}^{2}=\left\{\mathfrak{v} \in l_{h}^{2}, \mathfrak{v}_{0}=\mathfrak{v}_{N}=0\right\} .
$$

Given $\mathfrak{v} \in l_{h, 0}^{2}$ we introduce the basic (central) finite difference operators

$$
\begin{aligned}
\left(\delta_{x} \mathfrak{v}\right)_{j}=\frac{1}{2 h}\left(\mathfrak{v}_{j+1}-\mathfrak{v}_{j-1}\right), & 1 \leq j \leq N-1, \\
\left(\delta_{x}^{2} \mathfrak{v}\right)_{j}=\frac{1}{h^{2}}\left(\mathfrak{v}_{j+1}-2 \mathfrak{v}_{j}+\mathfrak{v}_{j-1}\right), & 1 \leq j \leq N-1 .
\end{aligned}
$$

The cornerstone of our approach to finite difference operators is the introduction of the Hermitian derivative of $\mathfrak{v} \in l_{h, 0}^{2}$, that will replace $\delta_{x}$. It will serve not only in approximating (to fourth-order of accuracy) first-order derivatives, but also as a fundamental building block in the construction of finite difference approximations to higher-order derivatives. First, we introduce the "Simpson operator"

$$
\left(\sigma_{x} \mathfrak{v}\right)_{j}=\frac{1}{6} \mathfrak{v}_{j-1}+\frac{2}{3} \mathfrak{v}_{j}+\frac{1}{6} \mathfrak{v}_{j+1}, \quad 1 \leq j \leq N-1 .
$$

Note the operator relation (valid in $l_{h, 0}^{2}$ )

$$
\sigma_{x}=I+\frac{h^{2}}{6} \delta_{x}^{2}
$$

so that $\sigma_{x}$ is an "approximation to identity" in the following sense. Let $\psi \in C_{0}^{\infty}(\Omega)$, then

$$
\left|\left(\sigma_{x}-I\right) \psi^{*}\right|_{\infty} \leq C h^{2}\left\|\psi^{\prime \prime}\right\|_{L^{\infty}(\Omega)}
$$

which yields

$$
\left|\left(\sigma_{x}-I\right) \psi^{*}\right|_{h} \leq C h^{2}\left\|\psi^{\prime \prime}\right\|_{L^{\infty}(\Omega)} .
$$

In the above estimates the constant $C>0$ is independent of $h, \psi$. The Hermitian derivative $\mathfrak{v}_{x}$ is now defined by

$$
\left(\sigma_{x} \mathfrak{v}_{x}\right)_{j}=\left(\delta_{x} \mathfrak{v}\right)_{j}, \quad 1 \leq j \leq N-1 .
$$


Remark 3.1. In the definition (3.9), the values of $\left(\mathfrak{v}_{x}\right)_{j}, j=0, N$, need to be provided, in order to make sense of the left-hand side (for $j=1, N-1$ ). If not otherwise specified, we shall henceforth assume that, in accordance with the boundary condition (1.4), $\mathfrak{v}_{x} \in l_{h, 0}^{2}$, namely

$$
\left(\mathfrak{v}_{x}\right)_{0}=\left(\mathfrak{v}_{x}\right)_{N}=0
$$

In particular, the linear correspondence $l_{h, 0}^{2} \ni \mathfrak{v} \rightarrow \mathfrak{v}_{x} \in l_{h, 0}^{2}$ is well defined, but not onto, since $\delta_{x}$ has a non-trivial kernel.

We next introduce a fourth-order replacement to the operator $\delta_{x}^{2}$ (see [17, Equation (15)], [6, Equation (10.50)(c)]),

$$
\left(\widetilde{\delta}_{x}^{2} \mathfrak{v}\right)_{j}=2\left(\delta_{x}^{2} \mathfrak{v}\right)_{j}-\left(\delta_{x} \mathfrak{v}_{x}\right)_{j}, \quad 1 \leq j \leq N-1 .
$$

The biharmonic discrete operator (DBO) is given by (for $\mathfrak{v}, \mathfrak{v}_{x} \in l_{h, 0}^{2}$ ),

$$
\delta_{x}^{4} \mathfrak{v}=\frac{12}{h^{2}}\left[\delta_{x} \mathfrak{v}_{x}-\delta_{x}^{2} \mathfrak{v}\right] .
$$

Note that, in accordance with Remark 3.1 the operator $\widetilde{\delta}_{x}^{2}$ is defined on grid functions $\mathfrak{v} \in l_{h, 0}^{2}$, such that also $\mathfrak{v}_{x} \in l_{h, 0}^{2}$. The connection between the two difference operators for the second-order derivative is given by

$$
-{\widetilde{\delta_{x}}}^{2}=-\delta_{x}^{2}+\frac{h^{2}}{12} \delta_{x}^{4} .
$$

Remark 3.2. Clearly the operators $\delta_{x}, \delta_{x}^{2}, \delta_{x}^{4}$ depend on $h$, but for notational simplicity this dependence is not explicitly indicated.

Remark 3.3. For clarity, the Taylor expansions of the operators used are collected in the Appendix in Section A.

The fact that the biharmonic discrete operator $\delta_{x}^{4}$ is positive (in particular symmetric) is proved in [6, Lemmas 10.9, 10.10]. Therefore its inverse $\left(\delta_{x}^{4}\right)^{-1}$ is also positive. Beyond just positivity, a fundamental tool (analogous to classical elliptic theory) is the coercivity property (with $C>0$ independent of $h$ ) [6, Propositions 10.11,10.13],

$$
\left(\delta_{x}^{4} \mathfrak{z}, \mathfrak{z}\right)_{h} \geq C\left(|\mathfrak{z}|_{h}^{2}+\left|\delta_{x}^{2} \mathfrak{z}\right|_{h}^{2}+\left|\delta_{x} \mathfrak{z} x\right|_{h}^{2}\right),
$$

valid for any grid function $\mathfrak{z} \in l_{h, 0}^{2}$ such that also $\mathfrak{z}_{x} \in l_{h, 0}^{2}$. Owing to (3.10) we can add also

$$
\left(\delta_{x}^{4} \mathfrak{z}, \mathfrak{z}\right)_{h} \geq C\left|\widetilde{\delta}_{x}^{2} \mathfrak{z}\right|_{h}^{2}
$$

Notation for time-dependent grid functions. A time-dependent grid function is denoted as $\left\{\mathfrak{v}\left(x_{j}, t\right)\right\}_{j=0}^{N}$ or $\left\{\mathfrak{v}_{j}(t)\right\}_{j=0}^{N}$. When there is no risk of confusion we simplify the notation, replacing $\left\{\mathfrak{v}\left(x_{j}, t\right)\right\}_{j=0}^{N},\left\{u^{*}\left(x_{j}, t\right)\right\}_{j=0}^{N}$ by $\mathfrak{v}(t), u^{*}(t)$ respectively.

\section{THE PURE BIHARMONIC GENERATOR}

We first establish convergence estimates for the simple equation

$$
\frac{\partial}{\partial t} u=-\left(\frac{\partial}{\partial x}\right)^{4} u, \quad x \in \Omega, t \geq 0
$$

subject to initial data

$$
u(x, 0)=u_{0}(x)
$$

and homogeneous boundary conditions

$$
u(0, t)=\frac{\partial}{\partial x} u(0, t)=u(1, t)=\frac{\partial}{\partial x} u(1, t)=0, \quad t \geq 0 .
$$

The initial function $u_{0}$ is assumed to be sufficiently smooth, and we take for simplicity $u_{0} \in C^{\infty}(\Omega)$. The semidiscrete analog of (4.1) is

$$
\frac{\partial}{\partial t} \mathfrak{v}\left(x_{j}, t\right)=-\delta_{x}^{4} \mathfrak{v}\left(x_{j}, t\right), \quad 1 \leq j \leq N-1, t \geq 0,
$$

subject to initial data

$$
\mathfrak{v}\left(x_{j}, 0\right)=\left(u_{0}^{*}\right)_{j}=u_{0}\left(x_{j}\right),
$$


and homogeneous boundary conditions

$$
\mathfrak{v}\left(x_{0}, t\right)=\mathfrak{v}_{x}\left(x_{0}, t\right)=\mathfrak{v}\left(x_{N}, t\right)=\mathfrak{v}_{x}\left(x_{N}, t\right)=0, \quad t \geq 0 .
$$

Here $\delta_{x}^{4}$ is the discrete biharmonic operator (3.11). Clearly the grid function $\mathfrak{v}(\cdot, t)$ depends on $h$, but we refrain from indicating it explicitly, as it will always be clear from the context (compare Remark 3.2). Occasionally the simplified notation $\mathfrak{v}(t)$ will be used instead of $\mathfrak{v}(\cdot, t)$, see the end of Section 3 . We recall the basic optimal convergence fact $[6$, Theorem 10.19]:

Claim 4.1. Let $f(x)$ be a smooth function in $\Omega$. Let $g(x)$ satisfy

$$
\left(\frac{d}{d x}\right)^{4} g(x)=f(x)
$$

subject to homogeneous boundary conditions. Then

$$
\left|g^{*}-\left(\delta_{x}^{4}\right)^{-1} f^{*}\right|_{\infty}=O\left(h^{4}\right)
$$

4.1. ALMOST OPTIMAL ERROR ESTIMATE-THE PURE BIHARMONIC CASE. We note that Equation (4.1) can be rewritten as

$$
\frac{\partial}{\partial t}\left(\frac{\partial}{\partial x}\right)^{-4} u=-u, \quad x \in \Omega, t \geq 0
$$

In view of Claim 4.1 (with $-\frac{\partial}{\partial t} u(\cdot, t)$ playing the role of $f$ and $u(\cdot, t)$ playing the role of $g$ ) we have, for any $T>0$, with $O\left(h^{4}\right)$ depending only on $u_{0}$ and $T$,

$$
\frac{\partial}{\partial t}\left(\delta_{x}^{4}\right)^{-1} u^{*}\left(x_{j}, t\right)=-u^{*}\left(x_{j}, t\right)+O\left(h^{4}\right), \quad 1 \leq j \leq N-1,0<t \leq T .
$$

Note that by definition

$$
\left(\frac{\partial}{\partial t} u\right)^{*}\left(x_{j}, t\right)=\frac{\partial}{\partial t} u^{*}\left(x_{j}, t\right), \quad 1 \leq j \leq N-1, t \geq 0
$$

Similarly, Equation (4.2) can be rewritten as

$$
\frac{\partial}{\partial t}\left(\delta_{x}^{4}\right)^{-1} \mathfrak{v}\left(x_{j}, t\right)=-\mathfrak{v}\left(x_{j}, t\right), \quad 1 \leq j \leq N-1, t \geq 0 .
$$

The smoothness of the initial data implies the boundedness of all time derivatives of the solution, as stated in the following claim.

Lemma 4.2. For every integer $N \geq 0$

$$
\left|\left(\frac{\partial}{\partial t}\right)^{N} \mathfrak{v}(\cdot, t)\right|_{h} \leq\left|\left(\delta_{x}^{4}\right)^{N} u_{0}^{*}\right|_{h}, \quad t \geq 0 .
$$

Proof. Taking the scalar product of $(4.2)$ with $\mathfrak{v}(\cdot, t)$ we have, due to the positivity of $\delta_{x}^{4}$,

$$
\frac{1}{2} \frac{\partial}{\partial t}|\mathfrak{v}(\cdot, t)|_{h}=-\left(\delta_{x}^{4} \mathfrak{v}(\cdot, t), \mathfrak{v}(\cdot, t)\right)_{h} \leq 0 .
$$

Thus $|\mathfrak{v}(\cdot, t)|_{h}$ is a nonincreasing function of $t$ and its value at $t=0$ is $\left|u_{0}^{*}\right|_{h}$. This proves the claim for $N=0$. We then proceed to all $N$ by repeated differentiation of (4.2) with respect to $t$, and noting that

$$
\left(\frac{\partial}{\partial t}\right)^{N} \mathfrak{v}(t=0)=\left(-\delta_{x}^{4}\right)^{N} u_{0}^{*}
$$

The difference between the exact solution and the discrete one is given by the "error" grid function

$$
\mathfrak{e}(t)=u^{*}(t)-\mathfrak{v}(t)
$$

The following theorem gives an estimate for this error.

Theorem 4.3. For every $\varepsilon>0$ and every $T>0$ the error can be estimated by

$$
|\mathfrak{e}(t)|_{h} \leq C h^{4-\varepsilon}, \quad t \in[0, T], h<h_{0},
$$

where $C>0$ depends only on $u_{0}, T, \varepsilon$. 
Proof. Define the grid function

$$
\mathfrak{w}(t)=\left(\delta_{x}^{4}\right)^{-1} \mathfrak{e}(t)=\left(\delta_{x}^{4}\right)^{-1} u^{*}(t)-\left(\delta_{x}^{4}\right)^{-1} \mathfrak{v}(t) .
$$

Subtracting Equation (4.6) from Equation (4.5) we obtain

$$
\mathfrak{w}^{\prime}(t)+\mathfrak{e}(t)=O\left(h^{4}\right), \quad t \in[0, T],
$$

with $\mathfrak{w}(0)=0$. Taking the discrete scalar product of (4.11) with $\mathfrak{w}$ and using the coercivity property (3.13), noting that $\mathfrak{e}(t)=\delta_{x}^{4} \mathfrak{w}(t)$, we get

$$
\frac{d}{d t}|\mathfrak{w}(t)|_{h}^{2}+C|\mathfrak{w}(t)|_{h}^{2} \leq\left|\left(O\left(h^{4}\right), \mathfrak{w}(t)\right)_{h}\right| \leq O\left(h^{8}\right)+\frac{C}{2}|\mathfrak{w}(t)|_{h}^{2}
$$

It follows from Gronwall's inequality that

$$
|\mathfrak{w}(t)|_{h}=O\left(h^{4}\right), \quad t \in[0, T] .
$$

Take an integer $Q>1$. We define a finite difference operator

$$
S_{Q} \mathfrak{w}(t)=(\Delta t)^{-1} \sum_{k=-L}^{M} a_{k} \mathfrak{w}(t+k \Delta t),
$$

so that

$$
\left|S_{Q} \mathfrak{w}(t)-\mathfrak{w}^{\prime}(t)\right|=O\left((\Delta t)^{Q}\right), \quad t \in[L \Delta t, T-M \Delta t]
$$

Observe that we can find such a difference operator, to any order, since all time derivatives of $\mathfrak{w}(t)$ are bounded in the $|\cdot|_{h}$ norm in view of the bounds (2.6), Lemma 4.2 and the uniform boundedness of the operator $\left(\delta_{x}^{4}\right)^{-1}$ for $h<h_{0}$. Note also that $M, L$ depend on $Q$. Plugging this into (4.11) we obtain

$$
S_{Q} \mathfrak{w}(t)+\mathfrak{e}(t)=O\left(h^{4}\right)+O\left((\Delta t)^{Q}\right), \quad t \in[L \Delta t, T-M \Delta t],
$$

and the estimate (4.13), applied to $S_{Q} \mathfrak{w}(t)$, yields

$$
\mathfrak{e}(t)=O\left(h^{4}\right)+O\left((\Delta t)^{Q}\right)+O\left(\frac{h^{4}}{\Delta t}\right), \quad t \in[L \Delta t, T-M \Delta t] .
$$

Take now $\Delta t=h^{\frac{4}{Q}}$. The last estimate yields

$$
\mathfrak{e}(t)=O\left(h^{4\left(1-\frac{1}{Q}\right)}\right), \quad t \in\left[L h^{\frac{4}{Q}}, T-M h^{\frac{4}{Q}}\right] .
$$

Since $Q$ can be taken arbitrarily large, the theorem is proved.

\section{A DISCRETE VERSION OF THE GENERAL EVOLUTION EQUATION}

Using the finite difference operators introduced in Section 3, and taking $h=\frac{1}{N}$, we introduce the discrete operator analogous to $\mathcal{L}_{A, B}$ that was defined in (1.1):

$$
\left[\mathcal{L}_{A, B, h} \mathfrak{g}\right]_{i}=\left(\delta_{x}^{4} \mathfrak{g}\right)_{i}-A_{i}^{*}\left(\widetilde{\delta}_{x}^{2} \mathfrak{g}\right)_{i}-\left(A^{\prime}\right)_{i}^{*}\left(\mathfrak{g}_{x}\right)_{i}+B_{i}^{*} \mathfrak{g}_{i}, \quad 1 \leq i \leq N-1,
$$

where $f^{*}, A^{*},\left(A^{\prime}\right)^{*}, B^{*}$ are the grid functions corresponding, respectively, to $f(x), A(x), A^{\prime}(x), B(x)$. In order to formulate a semi-discrete analog of (1.2), we introduce the unknown grid function $\mathfrak{v}\left(x_{j}, t\right)$, depending on the continuous time parameter. We do not indicate explicitly the obvious dependence on "h", since this will be clear from the context, as in Section 4. The semi-discrete equation is therefore

$$
\frac{\partial}{\partial t} \mathfrak{v}\left(x_{j}, t\right)=-\mathcal{L}_{A, B, h} \mathfrak{v}\left(x_{j}, t\right)+f^{*}\left(x_{j}\right), \quad 1 \leq j \leq N-1, t \geq 0
$$

subject to initial data

and homogeneous boundary conditions

$$
\mathfrak{v}\left(x_{j}, 0\right)=\left(u_{0}^{*}\right)_{j}=u_{0}\left(x_{j}\right)
$$

$$
\mathfrak{v}\left(x_{0}, t\right)=\mathfrak{v}_{x}\left(x_{0}, t\right)=\mathfrak{v}\left(x_{N}, t\right)=\mathfrak{v}_{x}\left(x_{N}, t\right)=0, \quad t \geq 0 .
$$

Remark 5.1. We assume that all grid functions and their Hermitian derivatives are in $l_{h, 0}^{2}$. This amounts simply to extending the grid functions (whose relevant values are at the interior points $\left\{x_{i}, 1 \leq i \leq N-1\right\}$ ) as zero at the endpoints $x_{0}, x_{N}$. 
In the proof of the convergence theorem below, an important ingredient is the coercivity of the operator, in analogy with Claim 2.1. It is actually a generalization of (3.13) that played a fundamental role in the case of the pure biharmonic generator.

Lemma 5.2. By adding to $B(x)$ a sufficiently large constant $K>0$, the following coercivity inequality holds.

There exist positive constants $C, h_{0}>0$ such that, for every grid function $\mathfrak{z} \in l_{h, 0}^{2}$, where also $\mathfrak{z} x \in l_{h, 0}^{2}$, we have

$$
\left(\mathcal{L}_{A, B, h \mathfrak{z}}, \mathfrak{z}\right)_{h} \geq C\left[\left|\widetilde{\delta}_{x}^{2} \mathfrak{z}\right|_{h}^{2}+\left|\mathfrak{z}_{x}\right|_{h}^{2}+|\mathfrak{z}|_{h}^{2}\right], \quad 0<h<h_{0}
$$

Proof. We have

$$
\begin{aligned}
\left(\mathcal{L}_{A, B, h \mathfrak{z}} \mathfrak{z}\right)_{h}= & \left(\delta_{x}^{4} \mathfrak{z}, \mathfrak{z}\right)_{h}-\left(A^{*} \widetilde{\delta}_{x}^{2} \mathfrak{z}, \mathfrak{z}\right)_{h}-\left(\left(A^{\prime}\right)^{*} \mathfrak{z} x, \mathfrak{z}\right)_{h}+\left(B^{*} \mathfrak{z}, \mathfrak{z}\right)_{h} \\
& \geq\left(\delta_{x}^{4} \mathfrak{z}, \mathfrak{z}\right)_{h}-\varepsilon\left[\left|\widetilde{\delta}_{x}^{2} \mathfrak{z}\right|_{h}^{2}+|\mathfrak{z} x|_{h}^{2}\right]-\frac{\beta}{\varepsilon}|\mathfrak{z}|_{h}^{2}+\left(B^{*} \mathfrak{z}, \mathfrak{z}\right)_{h},
\end{aligned}
$$

where we have used the Cauchy-Schwarz inequality and set $\beta=\left|A^{*}\right|_{\infty}^{2}+\left|\left(A^{\prime}\right)^{*}\right|_{\infty}^{2}$.

In view of (3.13) and (3.14) we can take $\varepsilon>0$ sufficiently small and then add a suitable constant $K>0$ to $B^{*}$ so that (5.3) is satisfied.

The basic convergence result here is that "stability" implies "convergence" as follows.

Theorem 5.3. [General convergence] Let $u(x, t)$ be the solution to $(1.2)$ and let $\mathfrak{v}\left(x_{j}, t\right)$ be the solution to $(5.2)$. Let

$$
\mathfrak{e}(t)=u^{*}(t)-\mathfrak{v}(t)
$$

(compare (4.8)). Then, for every $T>0$, we have the following convergence result:

$$
\lim _{h \rightarrow 0} \sup _{0 \leq t \leq T}|\mathfrak{e}(t)|_{h}=0 \text {. }
$$

Proof. The coercivity property stated in Lemma 5.2 implies, exactly as in the discussion prior to Claim 2.1 , that by adding a sufficiently large constant $c>0$ to $B(x)$, without changing notation, we can assume that $\mathcal{L}_{A, B, h}$ satisfies (5.3). Let $\tau(h)=o(h)$ be the truncation error arising in the application of the discrete operator $\mathcal{L}_{A, B, h}$ to the grid function $u^{*}\left(x_{j}, t\right)$, namely

$$
\frac{\partial}{\partial t} u^{*}\left(x_{j}, t\right)=-\mathcal{L}_{A, B, h} u^{*}\left(x_{j}, t\right)+f^{*}\left(x_{j}\right)+\tau(h), \quad 1 \leq j \leq N-1 .
$$

Observe that $\tau(h)$ is in fact also a function of $t$, such that for any $\varepsilon>0$ and $T>0$ there exists $h_{0}>0$ so that

$$
|\tau(h)|_{h} \leq \varepsilon h, \quad 0<h<h_{0}, 0 \leq t<T \text {. }
$$

Subtracting (5.2) from (5.5) we obtain (using the simplified notation for time dependent grid functions)

$$
\frac{\partial}{\partial t} \mathfrak{e}(t)=-\mathcal{L}_{A, B, h} \mathfrak{e}(t)+\tau(h)
$$

Taking the scalar product (in $l_{h, 0}^{2}$ ) of $(5.7)$ with $\mathfrak{e}(t)$ and using the coercivity inequality (5.3) yields

$$
\frac{1}{2} \frac{d}{d t}|\mathfrak{e}(t)|_{h}^{2} \leq-\frac{1}{2} C|\mathfrak{e}(t)|_{h}^{2}+\frac{2}{C}|\tau(h)|_{h}^{2},
$$

where the Cauchy-Schwarz unequality was used to estimate the scalar product $(\tau(h), \mathfrak{e}(t))_{h}$. In view of $(5.6)$ and Gronwall's inequality the proof is complete.

\section{THE LINEAR EVOLUTION EQUATION WITH CONSTANT COEFFICIENTS}

In Equation (1.2) we now consider the case $A(x) \equiv a, B(x) \equiv b, f \equiv 0$, where $a, b$ are real constants. Using the operator notation (compare (1.1))

the equation takes the form

$$
\mathcal{L}_{a, b} z=\left(\frac{d}{d x}\right)^{4} z-a\left(\frac{d}{d x}\right)^{2} z+b z
$$

$$
\frac{\partial}{\partial t} u(x, t)=-\mathcal{L}_{a, b} u(x, t), \quad x \in \Omega, t \geq 0 .
$$

The equation is supplemented with initial data

$$
u(x, 0)=u_{0}(x)
$$


and homogeneous boundary conditions

$$
u(0, t)=\frac{\partial}{\partial x} u(0, t)=u(1, t)=\frac{\partial}{\partial x} u(1, t)=0, \quad t \geq 0 .
$$

The initial function $u_{0}$ is assumed to be smooth, namely $u_{0} \in C^{\infty}(\Omega)$. As already observed above (see (2.1)) by taking the constant $b$ sufficiently large the coercivity can be assumed :

$$
\left(\mathcal{L}_{a, b} z, z\right)_{L^{2}(\Omega)} \geq C\left[\left\|z^{\prime \prime}\right\|_{L^{2}(\Omega)}^{2}+\left\|z^{\prime}\right\|_{L^{2}(\Omega)}^{2}+\|z\|_{L^{2}(\Omega)}^{2}\right] .
$$

Next we introduce the corresponding discrete operator (as in (5.1))

$$
\mathcal{L}_{a, b, h} \mathfrak{v}\left(x_{j}, t\right)=\delta_{x}^{4} \mathfrak{v}\left(x_{j}, t\right)-a \widetilde{\delta}_{x}^{2} \mathfrak{v}\left(x_{j}, t\right)+b \mathfrak{v}\left(x_{j}, t\right), 1 \leq j \leq N-1 .
$$

The coercivity in the general case (5.3) yields in this special case the following estimate (for $b>0$ sufficiently large).

$$
\left(\mathcal{L}_{a, b, h \mathfrak{z}}, \mathfrak{z}\right)_{h} \geq C\left[\left|\widetilde{\delta}_{x}^{2} \mathfrak{z}\right|_{h}^{2}+|\mathfrak{z} x|_{h}^{2}+|\mathfrak{z}|_{h}^{2}\right], \quad 0<h<h_{0},
$$

valid for every grid function $\mathfrak{z} \in l_{h, 0}^{2}$, such that also $\mathfrak{z} x \in l_{h, 0}^{2}$.

Claim 2.1 and (6.2) imply in particular that the operators $\mathcal{L}_{a, b}, \mathcal{L}_{a, b, h}$ are invertible and the norms $\left\{\left|\mathcal{L}_{a, b, h}^{-1}\right|_{h}, 0<h<h_{0}\right\}$ are uniformly bounded. Throughout the rest of this section we assume that $b>0$ is sufficiently large, as stipulated in these claims. The semi-discrete analog of (6.1) is

$$
\frac{\partial}{\partial t} \mathfrak{v}\left(x_{j}, t\right)=-\mathcal{L}_{a, b, h} \mathfrak{v}\left(x_{j}, t\right), \quad 1 \leq j \leq N-1, t \geq 0,
$$

subject to initial data

$$
\mathfrak{v}\left(x_{j}, 0\right)=\left(u_{0}^{*}\right)_{j}=u_{0}\left(x_{j}\right), \quad 1 \leq j \leq N-1 .
$$

and homogeneous boundary conditions

$$
\mathfrak{v}\left(x_{0}, t\right)=\mathfrak{v}_{x}\left(x_{0}, t\right)=\mathfrak{v}\left(x_{N}, t\right)=\mathfrak{v}_{x}\left(x_{N}, t\right)=0, \quad t \geq 0 .
$$

In analogy with (4.4) we can rewrite (1.2) as

$$
\frac{\partial}{\partial t}\left(\mathcal{L}_{a, b}^{-1} u(x, t)\right)=\mathcal{L}_{a, b}^{-1}\left(\frac{\partial}{\partial t} u(x, t)\right)=-u(x, t), \quad x \in \Omega, t \geq 0 .
$$

Similarly, the analog of (4.6) is

$$
\frac{\partial}{\partial t}\left(\mathcal{L}_{a, b, h}^{-1} \mathfrak{v}\left(x_{j}, t\right)\right)=\mathcal{L}_{a, b, h}^{-1}\left(\frac{\partial}{\partial t} \mathfrak{v}\left(x_{j}, t\right)\right)=-\mathfrak{v}\left(x_{j}, t\right), \quad 1 \leq j \leq N-1, t \geq 0
$$

The crucial element in our treatment is the "optimal" estimate for the (constant coefficient) elliptic case, which extends the same one in the pure biharmonic case (Claim 4.1).

Claim 6.1. [8, Theorem 5.7] Let $f(x)$ be a smooth function in $\Omega$. Let $g(x)$ satisfy

$$
\mathcal{L}_{a, b} g(x)=f(x),
$$

subject to homogeneous boundary conditions. Then

$$
\left|g^{*}-\mathcal{L}_{a, b, h}^{-1} f^{*}\right|_{\infty}=O\left(h^{4}\right) .
$$

We can now apply Claim 6.1 to Equation (6.4), where $\frac{\partial}{\partial t} u(x, t)$ corresponds to $f$, while $-u$ corresponds to $g$. We infer that

$$
\mathcal{L}_{a, b, h}^{-1}\left(\frac{\partial}{\partial t} u^{*}\left(x_{j}, t\right)\right)=-u^{*}\left(x_{j}, t\right)+O\left(h^{4}\right)
$$

Defining the error grid function $\mathfrak{e}(t)=u^{*}(t)-\mathfrak{v}(t)$ (compare (4.8)) we obtain, by subtracting (6.5) from (6.7)

$$
\frac{\partial}{\partial t} \mathcal{L}_{a, b, h}^{-1} \mathfrak{e}(t)=-\mathfrak{e}(t)+O\left(h^{4}\right) .
$$

The following theorem gives the "almost optimal" convergence estimate for the general equation and constitutes a generalization of Theorem 4.3. 
Theorem 6.2. Suppose that $u_{0} \in C^{\infty}(\Omega)$. For every $\varepsilon>0$ and every $T>0$ the error $\mathfrak{e}(t)$ can be estimated by

$$
|\mathfrak{e}(t)|_{h} \leq C h^{4-\varepsilon}, \quad t \in[0, T], h<h_{0},
$$

where $C>0$ depends only on $u_{0}, T, \varepsilon$.

Proof. Define the grid function

$$
\mathfrak{w}(t)=\mathcal{L}_{a, b, h}^{-1} \mathfrak{e}(t)=\mathcal{L}_{a, b, h}^{-1} u^{*}(t)-\mathcal{L}_{a, b, h}^{-1} \mathfrak{v}(t) .
$$

From Equation (6.8) we get

$$
\mathfrak{w}^{\prime}(t)+\mathfrak{e}(t)=O\left(h^{4}\right), \quad t \in[0, T],
$$

with $\mathfrak{w}(0)=0$. This equation is identical to Equation (4.11), so that the proof proceeds verbatim as that of Theorem 4.3. Note that instead of using the coercivity property (3.13), we use here the coercivity property (6.2).

\section{NUMERICAL EXAMPLES - LINEAR AND NONLINEAR}

Theorem 5.3 dealt with a general convergence result for linear equations with variable coefficients while Theorem 6.2 established an almost fourth-order accuracy of the semi-discrete scheme (6.3), in the case of constant coefficients. In this section, we first corroborate the assertion of Theorem 6.2 by four numerical test cases with constant coefficients. Indeed, the numerical results yield a full fourth-order accuracy. We then consider a linear equation with variable coefficients in the fifth test case. The excellent approximation (to known analytical solutions) achieved by employing the same difference operators is indicative of the applicability of the methodology developed here to more general evolution equations. Then, with the sixth and seventh test cases, we go beyond the linear setting that has been the subject matter of the general convergence theory expounded hitherto and demonstrate the effective fourth-order accuracy on several nonlinear biharmonic equations. This includes numerical solutions of the well-known Kuramoto-Sivashinsky equation, modelling the evolution of flame fronts (as well as other physical phenomena).

Discrete time stepping: Notice that in the convergence theory of the preceding sections we have used a "semi-discrete" scheme; the time variable was continuous. Numerical implementation necessitates discrete time intervals. Semi-discrete equations considered hereafter have the general form

$$
\partial_{t} \mathfrak{v}=-\delta_{x}^{4} \mathfrak{v}+L(\mathfrak{v})+H(\mathfrak{v})+F(t)=R(\mathfrak{v}, t),
$$

where $L$ is linear in $\mathfrak{v}, \mathfrak{v}_{x}, \tilde{\delta}_{x}^{2} \mathfrak{v}$ and $H$ is nonlinear is $\mathfrak{v}, \mathfrak{v}_{x} . F(t)=\left[F_{1}(t), \ldots, F_{N-1}(t)\right]$ is a time-dependent forcing function.

Two time stepping schemes are considered. The first one is the two stage Radau-IIA scheme. It is an IRK (Implicit Runge-Kutta) scheme, of third order accuracy, with A- and L-stability. Using this scheme allows to focus only on observing the accuracy in space. This is true in particular for linear problems. In the computation, due to the third order accuracy in time, the time step is selected according to a relation $\Delta t=C h^{4 / 3}$. The matrix $A$ of the Radau-IIA scheme is [12, chap. 3, pp. 225]

$$
A=\left[\begin{array}{l}
a_{11}, a_{12} \\
a_{21}, a_{22}
\end{array}\right]=\left[\begin{array}{cc}
\frac{5}{12} & -\frac{1}{12} \\
\frac{3}{4} & \frac{1}{4}
\end{array}\right] .
$$

The stage time values are $\Delta t / 3$ and $\Delta t$, respectively. The scheme is expressed as

$$
\left\{\begin{array}{l}
\xi_{1}=u^{n}+\Delta t\left(a_{11} R\left(\xi_{1}\right)+a_{12} R\left(\xi_{2}\right)\right), \\
\xi_{2}=u^{n}+\Delta t\left(a_{21} R\left(\xi_{1}\right)+a_{22} R\left(\xi_{2}\right)\right)
\end{array}\right.
$$

with $u^{n+1}=\xi^{2}$ (FSAL ${ }^{1}$ property). The system (7.3) is solved by Newton iterations. The second time stepping scheme is the IMEX (IMplicit-EXplicit) scheme already used in [3]. Here, it is used only for the KuramotoSivashinski equation (test cases 7 hereafter). As a rule, we denote by $u^{*, n}$ (see (3.1)) the grid values, at time level

\footnotetext{
${ }^{1}$ First Same As Last
} 
$t=t_{n}$, of the exact solution. The discrete solution at this time level is designated as $\mathfrak{v}^{n}$. The computational errors are designated as follows.

$$
\begin{aligned}
\left|\mathfrak{e}^{n}\right|_{h} & =\left|\mathfrak{v}^{n}-u^{*, n}\right|_{h} \text { the error (of the computed solution) in the discrete } l_{h}^{2} \text { norm, as in (3.2), } \\
\left|\mathfrak{e}_{x}^{n}\right|_{h} & =\left|\mathfrak{v}_{x}^{n}-\left(\frac{\partial u}{\partial x}\right)^{*, n}\right|_{h} \text { the error, using discrete } l_{h}^{2} \text { norm, for the } x \text { - derivative of the solution. }
\end{aligned}
$$

Note that $\mathfrak{v}_{x}^{n}$ is the Hermitian derivative (with respect to $x$ ) of $\mathfrak{v}^{n}$. However we compare to the exact derivative $\frac{\partial u}{\partial x}$ and not to the Hermitian derivative $\left(u^{*}\right)_{x}^{n}$. We shall also use the sup norm (see (3.3)) $\left|\mathfrak{e}^{n}\right|_{\infty}=\left|\mathfrak{v}^{n}-u^{*, n}\right|_{\infty}$. Recall that $e^{n}$, as a grid function, depends on $h$. To display the convergence rate, we make it explicit by wrrting $e_{h}^{n}$. Then the convergence rate between the error $\mathfrak{e}_{h}$ and $\mathfrak{e}_{h / 2}$ is calculated by $\log _{2}\left(\left|\mathfrak{e}_{h}\right| /\left|\mathfrak{e}_{h / 2}\right|\right)$.

7.1. First test case: periodic biharmonic equation. We consider on the interval $I=[0,2 \pi)$ the biharmonic equation

$$
\left\{\begin{aligned}
\frac{\partial}{\partial t} u & =-\left(\frac{\partial}{\partial x}\right)^{4} u, x \in I, \quad t \geq 0 \\
u(x, 0) & =u_{0}(x)=\sin (x), \quad x \in I .
\end{aligned}\right.
$$

When periodic boundary conditions are applied, the solution is $u(x, t)=\sin (x) \exp (-t)$. The semi-discrete scheme is

$$
\left\{\begin{aligned}
\frac{d \mathfrak{v}_{j}(t)}{d t} & =-\delta_{x}^{4} \mathfrak{v}_{j}(t), \quad j=0,1, \ldots, N-1 \\
\mathfrak{v}_{N}(t) & =\mathfrak{v}_{0}(t), \quad t \geq 0 \\
\mathfrak{v}_{j}(0) & =\left(u_{0}^{*}\right)_{j}:=u_{0}\left(x_{j}\right), \quad j=0, \ldots, N
\end{aligned}\right.
$$

The discrete time-stepping is the Radau-IIA time scheme (7.3). The periodic boundary conditions make this test case easier to implement compared to the case of homogeneous boundary conditions. However, it enables us to focus on the spatial accuracy of the discrete biharmonic operator $\delta_{x}^{4}$. In Table 1, the accuracy of the scheme is compared to the piecewise cubic discontinuous Galerkin method of [32] (without local postprocessing). Both schemes are fourth-order accurate in space. The error levels are of the same order of magnitude for the two cases.

7.2. Second test case: polynomial initial data. We consider the equation

$$
\left\{\begin{array}{r}
\frac{\partial}{\partial t} u=-\left(\frac{\partial}{\partial x}\right)^{4} u+a\left(\frac{\partial}{\partial x}\right)^{2} u-b u+f(x, t), \quad 0<x<1, \quad t \geq 0 \\
u(0, t)=\frac{\partial}{\partial x} u(0, t)=0, \quad u(1, t)=\frac{\partial}{\partial x} u(1, t)=0, \quad t \geq 0 \\
u(x, 0)=u_{0}(x), \quad 0 \leq x \leq 1 .
\end{array}\right.
$$

The semi-discrete analog to (7.8) is (compare (6.3))

$$
\left\{\begin{aligned}
\frac{d \mathfrak{v}_{j}(t)}{d t} & =-\delta_{x}^{4} \mathfrak{v}_{j}(t)+a \tilde{\delta}_{x}^{2} \mathfrak{v}_{j}(t)-b \mathfrak{v}_{j}(t)+f_{j}^{*}(t), \quad j=1, \ldots, N-1, \quad t \geq 0, \\
\mathfrak{v}_{0}(t) & =0, \mathfrak{v}_{N}(t)=0, \mathfrak{v}_{x, 0}(t)=0, \mathfrak{v}_{x, N}(t)=0, \quad t \geq 0 \\
\mathfrak{v}_{j}(0) & =\left(u_{0}^{*}\right)_{j}:=u_{0}\left(x_{j}\right), \quad j=0, \ldots, N .
\end{aligned}\right.
$$

We picked $a=1, b=-1$ in $(7.8)$ with exact solution $u(x, t)=x^{4}(1-x)^{4} e^{t}$. The initial condition is therefore $u_{0}(x)=x^{4}(1-x)^{4}$. The function $f(x, t)$ is chosen so that (7.8) is satisfied. Note that in Claim 2.1 it was required that $b>0$ in order to ensure coercivity, and in particular that zero is not an eigenvalue of $\mathcal{L}_{a, b, h}$. However, for any value of $b \in \mathbf{R}$, if we know that zero is not an eigenvalue, then the discrete scheme has the "almost optimal" rate of convergence. Indeed, coercivity is restored (without changing the scheme) by multiplying the discrete equation by a suitable function $e^{c t}$ (see the beginning of the proof of Theorem 5.3). Several runs were performed with successively refined meshes. Due to third-order accuracy of the Radau-IIA scheme, we picked $\Delta t=K h^{4 / 3}$, where 
the constant $K$ is adjusted so that 8 iterations are performed for $N=8$. The numerical results are displayed in Table 2 and Figure 1.
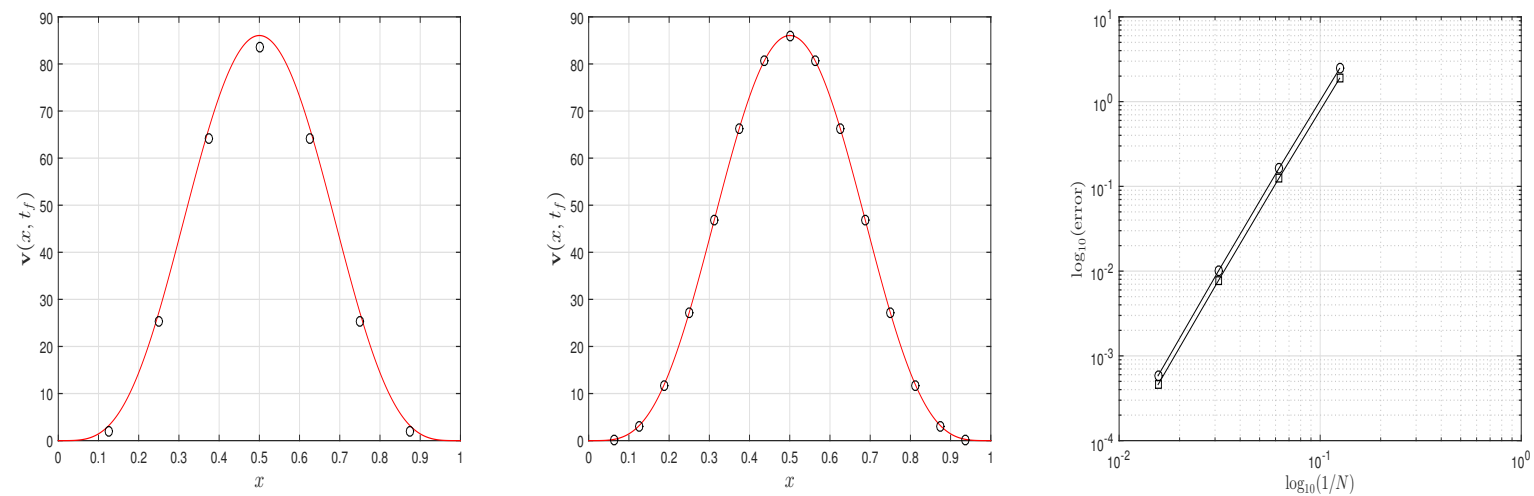

Figure 1. Exact and calculated solution for (7.8) with $a=1, b=-1$ and $u(x, t)=x^{4}(1-x)^{4} e^{t}$ at final time $t_{f}=10$. Left: $N=8$, center: $N=16$, right: the estimated convergence rate in the maximum (circles) and $l_{h}^{2}$ (squares) norms is 4 . Radau-IIA time scheme with $\Delta t=K h^{4 / 3}$. There are 8 time interations with the grid $N=*$. The error level at final time is excellent, even on the coarse grid $N=8$.

7.3. Third test case: highly oscillating solution. We consider again Equation (7.8), but now the exact solution is

$$
u_{\varepsilon}(x, t)=p(x) \sin \left(1 / q_{\varepsilon}(x)\right) \sin (2 \pi t) .
$$

The polynomial functions $p(x)$ and $q_{\varepsilon}(x)$ are given by

$$
p(x)=16 x^{2}(1-x)^{2}, \quad q_{\varepsilon}(x)=(x-1 / 2)^{2}+\varepsilon, \quad \varepsilon>0 .
$$

The parameter $\varepsilon$ has to be adjusted so that the function $u_{\varepsilon}(x, t)$ oscillates faster in the middle of the interval [0,1]. In this example we took $\varepsilon=0.05$. Numerical results using the Radau-IIA time scheme are displayed in Table 3 and Figure 2. The results, even for the very coarse grid with $N=32$, show the remarkable accuracy of the scheme.
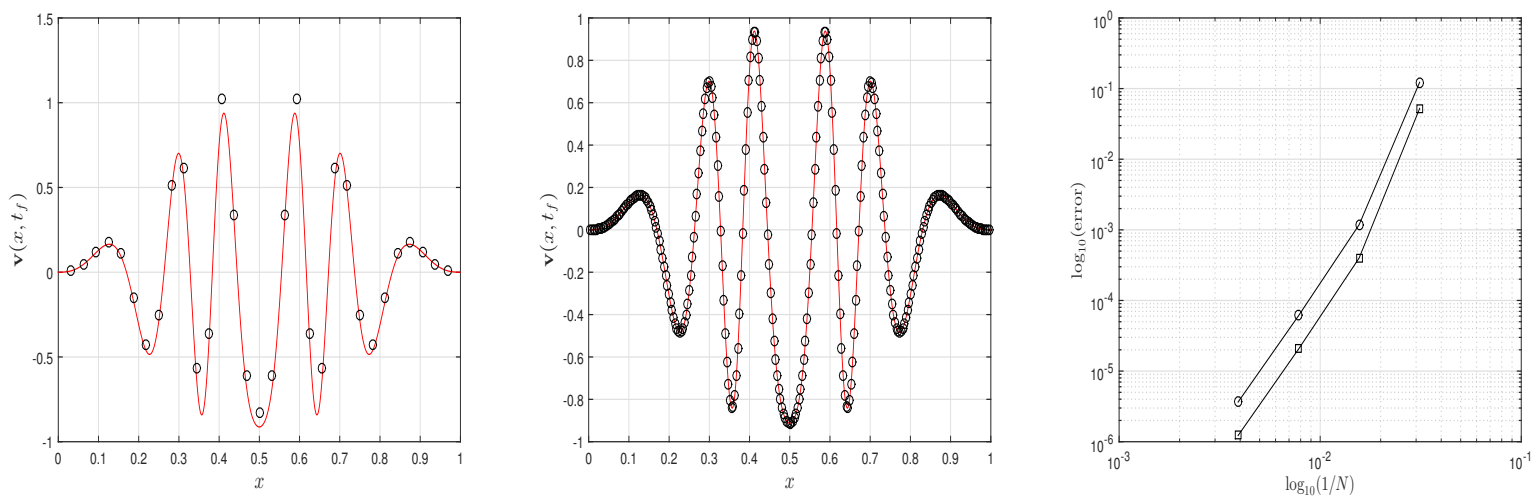

FiguRE 2. Exact and calculated solution $u(x, t)=p(x) \sin \left(1 / q_{\varepsilon}(x)\right) \sin (2 \pi t)$ for Equation (7.8) $\varepsilon=0.05$, at final time $t_{f}=0.75$. The number $N$ of grid points is $N=32, N=64, N=128$ and $N=256$. The convergence rate is indicated at final time $t_{f}=0.75$. Left: $N=32$, center: $N=256$, right: the observed convergence rate is close to 4 in the max norm (circles) and the $l_{h}^{2}$ norm (squares). Radau-IIA time scheme with $\Delta t=K h^{4 / 3}$. There are 10 time interations with the grid $N=32$. Notice the good error level, even on the coarse grid $N=32$, with 10 time iterations. 
7.4. Fourth test case: linear equation with spectral damped solution. We use this example to assess the difference between the discrete operators $\delta_{x}^{4},\left(\delta_{x}^{2}\right)^{2}$, both of which serve to approximate the fourth-order derivative. Let $\varphi_{k}(x)$ (resp. $\mu_{k}$ ) be the $k$-th eigenfunction (resp. $k$-th eigenvalue) of the spectral problem

$$
\left(\frac{d}{d x}\right)^{4} \varphi(x)=\mu \varphi(x)
$$

with homogeneous boundary conditions

$$
\varphi(0)=\frac{d}{d x} \varphi(0)=0, \quad \varphi(1)=\frac{d}{d x} \varphi(1)=0 .
$$

The eigenfunctions are analytically known, see for example [15, 18]. We consider the time dependent problem

$$
\left\{\begin{aligned}
\frac{\partial}{\partial t} u(x, t) & =-\left(\frac{\partial}{\partial x}\right)^{4} u(x, t)+\left(\mu_{k}-\frac{1}{\tau}\right) u(x, t), \quad 0<x<1, \quad t \geq 0, \\
u(0, t) & =0, \quad \frac{\partial}{\partial x} u(0, t)=0, \quad t \geq 0 \\
u(1, t) & =0, \quad \frac{\partial}{\partial x} u(1, t)=0, \quad t \geq 0 \\
u(x, 0) & =\varphi_{k}(x), \quad k \geq 1, \quad 0 \leq x \leq 1 .
\end{aligned}\right.
$$

Here $\tau>0$ is a parameter. The exact solution is given by the damping in time of the initial function $\varphi_{k}(x)$,

$$
u(x, t)=\varphi_{k}(x) \exp (-t / \tau)
$$

The aim here is to recover a similar behavior for the discrete solution. With the notation of Section 6 we have

$$
a=0, \quad b=-\left(\mu_{k}-1 / \tau\right), \quad f(x, t)=0 .
$$

In the numerical example we took $k=10$ and $\tau=\frac{10}{\mu_{k}}$, leading to $b=-\frac{9}{10} \mu_{k}$.

We use this example also to consider the difference between two semi-discrete schemes for (7.14). The first consists of (see (5.2)):

$$
\left\{\begin{aligned}
\frac{d \mathfrak{v}_{j}(t)}{d t} & =-\delta_{x}^{4} \mathfrak{v}_{j}(t)+\left(\mu_{k}-\frac{1}{\tau}\right) \mathfrak{v}_{j}(t), \quad j=1, \ldots, N-1, \quad t \geq 0 \\
\mathfrak{v}_{0}(t) & =0, \mathfrak{v}_{N}(t)=0, \quad t \geq 0 \\
\mathfrak{v}_{x, 0}(t) & =0, \mathfrak{v}_{x, N}(t)=0, \quad t \geq 0 \\
\mathfrak{v}_{j}(0) & =\varphi_{k, j}^{*}:=\varphi_{k}\left(x_{j}\right)
\end{aligned}\right.
$$

The second scheme replaces the compact $\delta_{x}^{4}$ by the standard five-point discrete biharmonic given in [10]:

$$
\left(\delta_{x}^{2}\right)^{2} \mathfrak{v}_{j}=\frac{\mathfrak{v}_{j+2}-4 \mathfrak{v}_{j+1}+6 \mathfrak{v}_{j}-4 \mathfrak{v}_{j-1}+\mathfrak{v}_{j-2}}{h^{4}}, \quad j=2, \ldots, N-2 .
$$

This scheme needs to be supplemented by an appropriate meaning for $\left(\delta_{x}^{2}\right)^{2} \mathfrak{v}_{j}$ at the near-boundary points $j=$ $1, N-1$, taking into account the Dirichlet boundary conditions as in (7.17). In [10] this is accomplished by a quadratic extrapolation based on the value at the near-boundary point; for $j=1$ the imposed boundary values $\mathfrak{v}_{0}=\mathfrak{v}_{x, 0}=0$ are used, along with the value $\mathfrak{v}_{1}$, obtaining an extrapolated value at the "ghost" point $j=-1$. Using this extrapolation approach leads to a formula for $\left(\delta_{x}^{2}\right)^{2} \mathfrak{v}_{1}$ given by

$$
\left(\delta_{x}^{2}\right)^{2} \mathfrak{v}_{1}=\frac{7 \mathfrak{v}_{1}-4 \mathfrak{v}_{2}+\mathfrak{v}_{3}}{h^{4}} .
$$




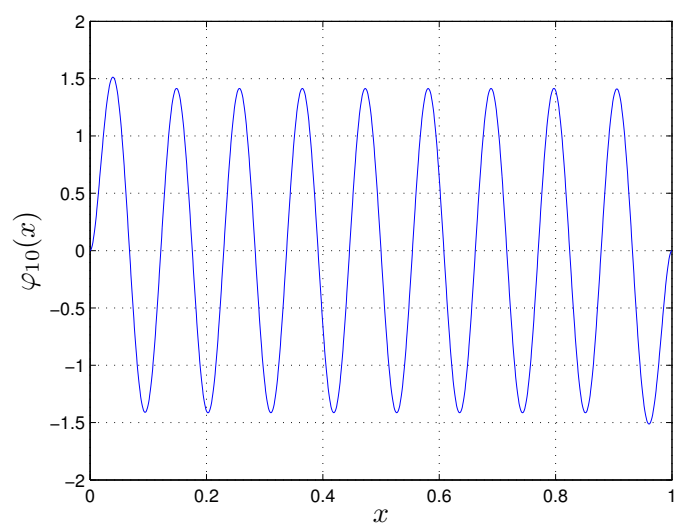

Figure 3. The eigenfunction $\varphi_{10}$ of the spectral problem (7.12) associated to the eigenvalue $\mu_{10}$.

A similar approximation is applied at $j=N-1$. The operator $\left(\delta_{x}^{2}\right)^{2}$ is second order at points $2 \leq j \leq N-2$. The associated semi-discrete scheme is

$$
\left\{\begin{aligned}
\frac{d \mathfrak{v}_{j}(t)}{d t} & =-\left(\delta_{x}^{2}\right)^{2} \mathfrak{v}_{j}(t)+\left(\mu_{k}-\frac{1}{\tau}\right) \mathfrak{v}_{j}(t), \quad j=1, \ldots, N-1, \quad t>0, \\
\mathfrak{v}_{0}(t) & =0, \mathfrak{v}_{N}(t)=0, \quad t \geq 0, \\
\mathfrak{v}_{x, 0}(t) & =0, \mathfrak{v}_{x, N}(t)=0, \quad t \geq 0, \\
\mathfrak{v}_{j}(0) & =\varphi_{k, j}^{*}:=\varphi_{k}\left(x_{j}\right), \quad j=1, \ldots, N-1,
\end{aligned}\right.
$$

Fig. 3 displays the oscillating shape of the eigenfunction $\varphi_{10}$. Note that $\varphi_{10}$ is antisymmetric with respect to the point $x=\frac{1}{2}$. The corresponding eigenvalue is $\mu_{10} \simeq 11,410,019.97$. The parameter $\tau$ represents a damping effect in time. The selected value is $\tau=\frac{10}{\mu_{10}}$. The grids are $N=8,16,32,64$ and $N=128$. The final time is $t_{f}=1 / \mu_{10}$. Fig. 4 represents the numerical convergence history for the schemes (7.17) and (7.20). In Figs. 5 and 6 the results of the schemes (7.17) and (7.20) are compared at final time for $N=32$ and $N=64$. The superiority of the operator $\delta_{x}^{4}$ over the operator $\left(\delta_{x}^{2}\right)^{2}$ is clearly observed: with $N=32$ the scheme (7.17) is more accurate than the scheme (7.20) with $N=64$.

7.5. Fifth test case: linear equation with non constant $A(x)$ and $B(x)$. We consider the function

$$
u(x, t)=U(x) \exp (\omega t), \quad U(x)=\exp \left(-\frac{1}{x(1-x)}\right) .
$$

The graph of $U(x)$ is displayed in Fig. 7. Note that $U(0)=U(1)=U^{\prime}(0)=U^{\prime}(1)=0$. The derivatives of $U(x)$ are given by

$$
U^{(n)}(x)=\frac{p_{n}(x)}{q_{n}(x)} U(x)
$$

The polynomials $p_{1}(x)$ and $q_{1}(x)$ are

$$
p_{1}(x)=1-2 x, \quad q_{1}(x)=x^{2}(1-x)^{2} .
$$

For $n \geq 2$, the following recursion relations hold

$$
\left\{\begin{array}{l}
p_{n+1}=q_{1}\left(p_{n}^{\prime} q_{n}-q_{n}^{\prime} p_{n}\right)+q_{n} p_{n} p_{1}, \\
q_{n+1}=q_{n}^{2} q_{1}
\end{array}\right.
$$

The polynomial $q_{n}$ is $q_{n}=q_{1}^{2^{n}-1}$. We consider the operator $\mathcal{L}_{A, B}(1.1)$ applied to $u(x, t)$, where $A(x)$ and $B(x)$ are selected below in (7.29) and (7.27) respectively. Recall that

$$
\mathcal{L}_{A, B} u(x, t)=\partial_{x}^{4} u(x, t)-\partial_{x}\left(A(x) \partial_{x} u(x, t)\right)+B(x) u(x, t) .
$$




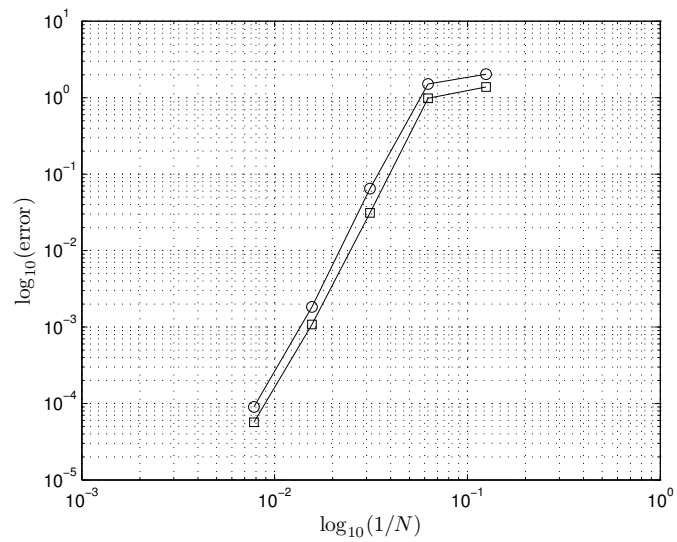

(a) Convergence rate for the scheme (7.17) at final time $t_{f}=$ $1 / \mu_{10}$ after time-stepping.

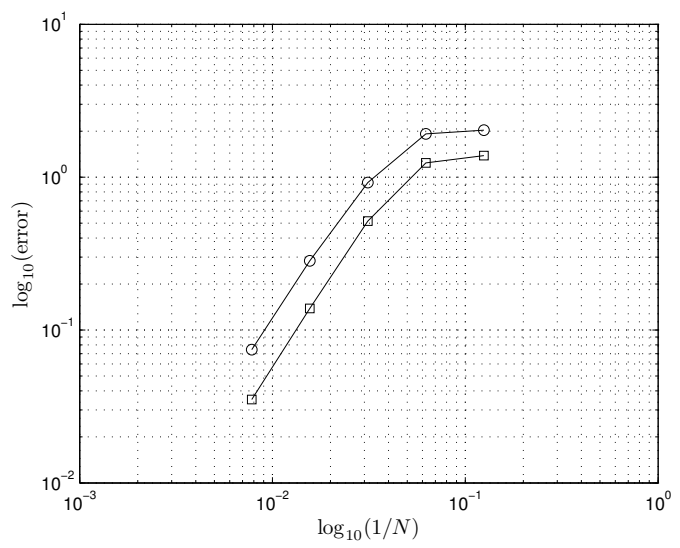

(b) Convergence rate for the scheme (7.20) at final time $t_{f}=$ $1 / \mu_{10}$ after time-stepping.

Figure 4. Convergence rates for the discrete approximations to the time dependent equation (7.14). (a): convergence rates for (7.17). (b): convergence rates for (7.20). For both subfigures: $N=8, N=16, N=32, N=64$ and $N=128$. (a): fourth order convergence rates with excellent error values for $\delta_{x}^{4}$. (b): second order convergence rates with poor accuracy for $\left(\delta_{x}^{2}\right)^{2}$.

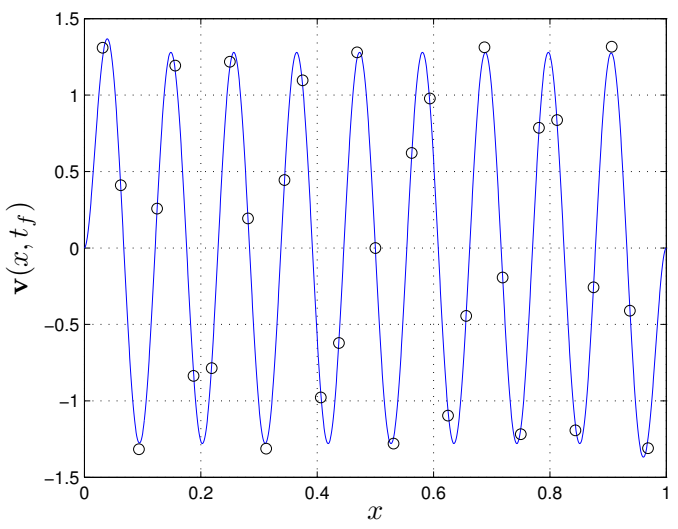

(a) Scheme (7.17) with $\delta_{x}^{4}$ and the grid $N=32$

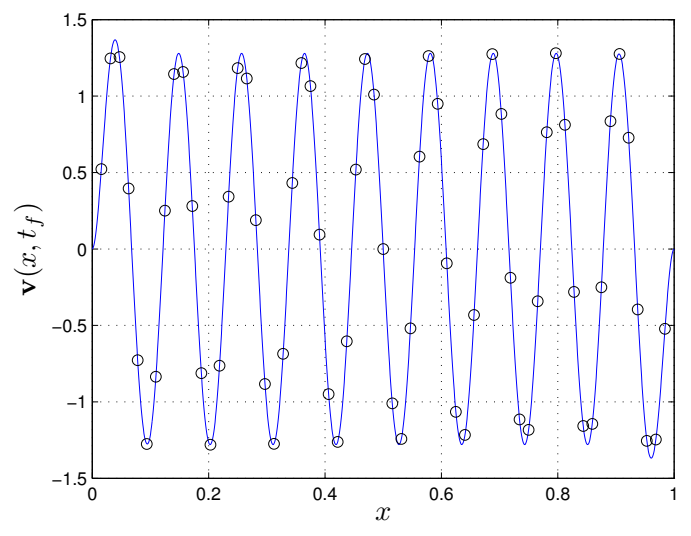

(b) Scheme (7.17) with $\delta_{x}^{4}$ and the grid $N=64$

Figure 5. Approximate and exact solution at final time $t_{f}=1 / \mu_{10}$. The equation (7.14) is approximated by (7.17). The biharmonic operator is approximated by the fourth order operator $\delta_{x}^{4}$. In the left panel, the grid size is $N=32$. In the right panel, the grid size is $N=64$. In both cases, the approximate solution is remarkably close to the exact one.

Using (7.24) we get

$$
\begin{aligned}
\left(\partial_{t}+\mathcal{L}_{A, B}\right) u(x, t) & =\omega U(x) \exp (\omega t)+\frac{p_{4}(x)}{q_{4}(x)} U(x) \exp (\omega t) \\
& -\partial_{x}\left(A(x) \frac{p_{1}(x)}{q_{1}(x)} U(x)\right) \exp (\omega t)+B(x) U(x) \exp (\omega t) .
\end{aligned}
$$




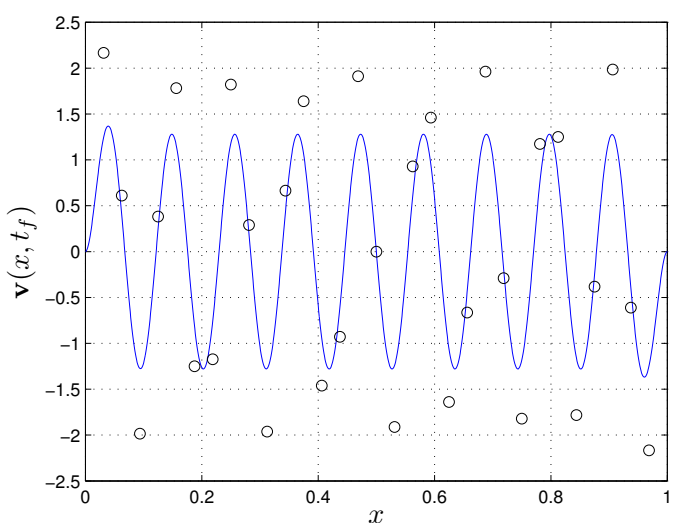

(a) Scheme (7.20) with $\left(\delta_{x}^{2}\right)^{2}, N=32$

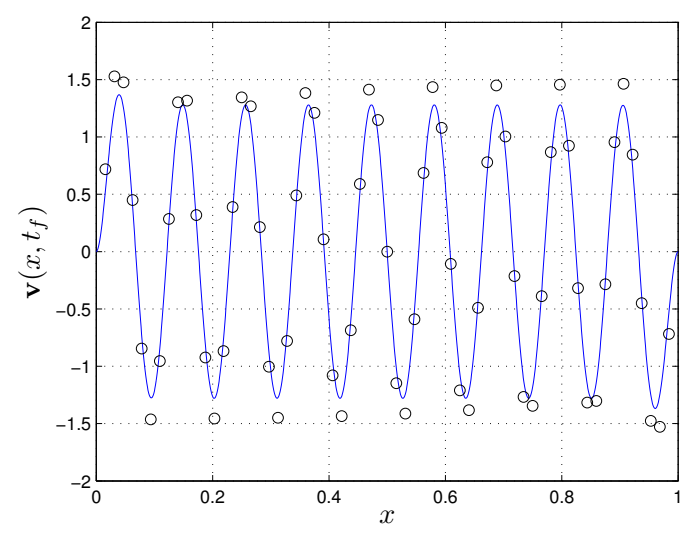

(b) Scheme (7.20) with $\left(\delta_{x}^{2}\right)^{2}, N=64$

Figure 6. Approximate and exact solution at final time $t_{f}=1 / \mu_{10}$. The equation (7.14) is approximated by (7.20). The biharmonic operator is approximated by the second order operator $\left(\delta_{x}^{2}\right)^{2}$. In the left panel, the grid size is $N=32$. In the right panel, the grid size is $N=64$. In both cases, the approximate solution is far from the exact one.

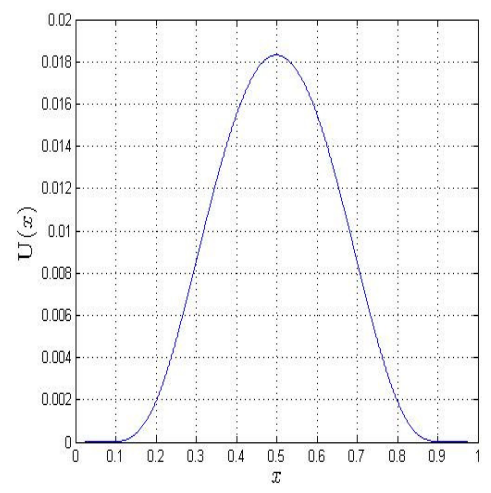

Figure 7 . The function $U(x)=\exp \left(-\frac{1}{x(1-x)}\right)$.

Given a function $A(x)$, if we define the function $B(x)$ to be

$$
\begin{aligned}
B(x) & =-\omega-\frac{p_{4}(x)}{q_{4}(x)}+A^{\prime}(x) \frac{p_{1}(x)}{q_{1}(x)}+A(x)\left(\frac{p_{1}(x)}{q_{1}(x)}\right)^{\prime}+A(x)\left(\frac{p_{1}(x)}{q_{1}(x)}\right)^{2} \\
& =-\omega-\frac{p_{4}(x)}{q_{4}(x)}+A^{\prime}(x) \frac{p_{1}(x)}{q_{1}(x)}+A(x)\left(\frac{p_{2}(x)}{q_{2}(x)}\right) .
\end{aligned}
$$

we see readily that $u(x, t)$ is the solution of the equation

$$
\partial_{t} u(x, t)=-\mathcal{L}_{A, B} u(x, t) .
$$

We take

$$
A(x)=1+0.1 \sin (2 \pi k x),
$$

and the corresponding function $B(x)$ is determined by (7.27). We then consider Equation (7.28) with the initial condition $u(x, 0)=U(x)$ and and the semi-discrete approximation (see (5.2))

$$
\partial_{t} \mathfrak{v}(t)=-\mathcal{L}_{A, B, h} \mathfrak{v}(t)
$$



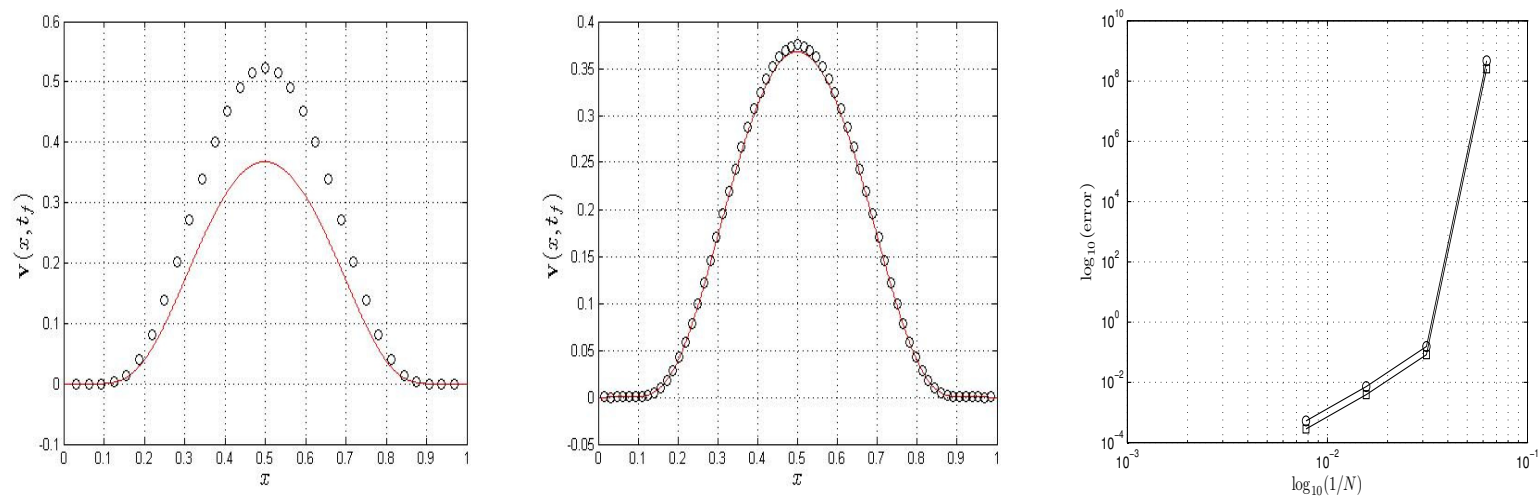

Figure 8. Equation (7.28) approximated by (7.30) with $A$ in (7.29) and $B$ in (7.27). The initial time is $t_{i}=0$. Results are shown at final time $t_{f}=3$. Left panel: $N=32$, the convergence in space is not achieved. Center panel: $N=64$, the convergence in space is achieved. Right panel: convergence rate in the maximum (circles) and $l_{h}^{2}$ (squares) norms) based on the values obtained for $N=16, N=32, N=64$ and $N=128$. The convergence rate in the lower part is close to 4 .

where the operator $\mathcal{L}_{A, B, h}$ is given by (5.1). The approximate solution of (7.30) is compared to the analytical solution $u(x, t)=U(x) \exp (\omega t)$. In the following we have chosen $k=10$ and $\omega=1$. In Fig. 8 we display the solution of the semi-discrete equation at final time $t=t_{f}$ after time-stepping using the grids $N=32$ and $N=64$. This solution is compared with the exact solution $u\left(x, t_{f}\right)=U(x) \exp \left(\omega t_{f}\right)$. We also record the convergence rate, adding a coarser $N=16$ grid and a finer one $N=128$. Note that there is a delicate interaction between the three finite difference operators $\delta_{x}^{4} \mathfrak{v}, \tilde{\delta}_{x}^{2} \mathfrak{v}$ and the Hermitian derivative $\mathfrak{v}_{x}$.

7.6. Sixth test case: a non linear example. A nonlinear modification of (7.8) is the evolution equation

$$
\left\{\begin{array}{r}
\frac{\partial}{\partial t} u=-\left(\frac{\partial}{\partial x}\right)^{4} u+H(u(x, t))+f(x, t), \quad 0<x<1, \\
u(0, t)=\frac{\partial}{\partial x} u(0, t)=0, \quad u(1, t)=\frac{\partial}{\partial x} u(1, t)=0 \\
u(x, 0)=u_{0}(x) .
\end{array}\right.
$$

The function $f(x, t)$ is selected so that the exact solution is $u_{\varepsilon}(x, t)$ with $\varepsilon=0.05$ (see (7.10)). Here $H(u)=$ $100 \sin ^{2} u$. The initial data is $u_{0}(x)=u_{\varepsilon}(x, 0)$. The analogous semi-discrete equation is

$$
\frac{d}{d t} \mathfrak{v}_{j}(t)=-\left(\delta_{x}^{4} \mathfrak{v}\right)_{j}+H\left(\mathfrak{v}_{j}(t)\right)+f^{*}\left(x_{j}, t\right), \quad 1 \leq j \leq N-1
$$

The time discretization is performed with the Radau-IIA scheme. The numerical results are displayed in Table 4 and Figure 9.

7.7. Seventh test case : Kuramoto-Sivashinsky equation. Consider the Kuramoto-Sivashinsky equation $[28,25]$

$$
\left\{\begin{aligned}
\frac{\partial}{\partial t} u & =-\left(\frac{\partial}{\partial x}\right)^{4} u-\left(\frac{\partial}{\partial x}\right)^{2} u-u \frac{\partial}{\partial x} u+f, \quad 0<x<1, \quad t>0 \\
u(0, t) & =0, \quad \frac{\partial}{\partial x} u(0, t)=0, \\
u(1, t) & =0, \quad \frac{\partial}{\partial x} u(1, t)=0 .
\end{aligned}\right.
$$

This equation has been independently derived in the context of several extended physical systems driven far from equilibrium by intrinsic instabilities, including instabilities of dissipative trapped ion modes in plasmas, instabilities in laminar flame fronts [28], phase dynamics in reaction-diffusion systems [25], and fluctuations in fluid 

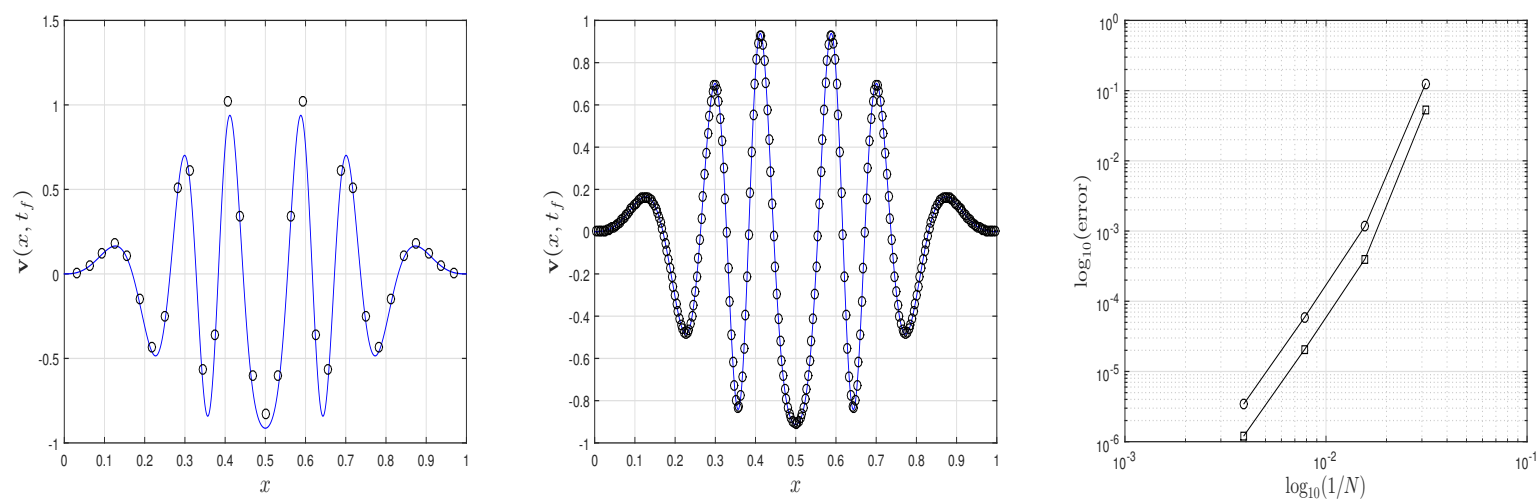

Figure 9. Nonlinear equation (7.31) with exact and calculated solution $u_{\varepsilon}(x, t)=$ $p(x) 1 / q_{\varepsilon}(x) \sin (2 \pi t), \varepsilon=0.05$, at final time $t_{f}=0.75$. The grids contains $N=32, N=64$, $N=128$ and $N=256$ points. Left panel: $N=32$, center panel: $N=256$, right panel: convergence rate in the maximum (circles) and $l_{h}^{2}$ (squares) norms. The rate is 4 . The time scheme is the Radau-IIA scheme with a time step $\Delta t=K h^{4 / 3}$ and 10 time iterations for $N=32$.

films on inclines [29]. The equation generically describes the dynamics near long-wave-length primary instabilities in the presence of appropriate (translational, parity and Galilean) symmetries. We present computations of three numerical examples, where the exact solutions are known and the error of the approximate solutions (and their derivatives) can be precisely measured. In the third example, there is no forcing function and the evolving solution develops steep slopes.

(1) First numerical example. We take $u(x, t)$ as

$$
u(x, t)=e^{-t} x^{2}(1-x)^{2}, \quad 0<x<1, \quad t>0,
$$

so that Equation (7.33) is satisfied with $f(x, t)$ taken accordingly. As in the previous test cases, we obtain the approximate solutions $\mathfrak{v}_{j}(t)$ of $u\left(x_{j}, t\right)$ from the knowledge of the discrete data $f_{j}^{*}(t)=f\left(x_{j}, t\right)$ on the grid $0=x_{0}<\cdots<x_{j}<x_{N}=1$. The approximate solutions are computed at discrete time levels $t_{n}=n \Delta t$, using a temporal discretization that is analogous to the second-order IMEX time-scheme that has been used in $[3,5]$.

$$
\left\{\begin{array}{l}
\frac{\mathfrak{v}_{j}^{n+1 / 2}-\mathfrak{v}_{j}^{n}}{\Delta t / 2}+\frac{1}{2}\left(\delta_{x}^{4} \mathfrak{v}_{j}^{n+1 / 2}+\tilde{\delta}_{x}^{2} \mathfrak{v}_{j}^{n+1 / 2}\right)=-\frac{1}{2}\left(\delta_{x}^{4} \mathfrak{v}_{j}^{n}+\tilde{\delta}_{x}^{2} \mathfrak{v}_{j}^{n}\right)-\mathfrak{v}_{j}^{n} \mathfrak{v}_{x, j}^{n}+f_{j}^{*, n}, \\
\frac{\mathfrak{v}_{j}^{n+1}-\mathfrak{v}_{j}^{n}}{\Delta t}+\frac{1}{2}\left(\delta_{x}^{4} \mathfrak{v}_{j}^{n+1}+\tilde{\delta}_{x}^{2} \mathfrak{v}_{j}^{n+1}\right)=-\frac{1}{2}\left(\delta_{x}^{4} \mathfrak{v}_{j}^{n}+\tilde{\delta}_{x}^{2} \mathfrak{v}_{j}^{n}\right)+\mathfrak{v}_{j}^{n+1 / 2} \mathfrak{v}_{x, j}^{n+1 / 2}+f_{j}^{*, n+1 / 2},
\end{array}\right.
$$

In Table 5 we display numerical results for (7.35). We have picked $\Delta t=h^{2}$, where $h=1 / N$, and the final time is $t_{f}=0.25$. Observe that the scheme (7.35) achieves fourth-order accuracy for $u$ and $\frac{\partial u}{\partial x}$. In Figure 10 we display the exact solution $u(x, t)$ (solid line) at $t=0.25$ and the computed solution $\mathfrak{v}$ (circles) for $N=32$ (left) and $N=64$ (center). The convergence rates are documented in the right panel. Notice the fourth-order rates both for $u$ and $\frac{\partial}{\partial x} u$. Notice also that even with a coarse mesh $N=16$ the match is excellent.

(2) Second numerical example. We consider again the Kuramoto-Sivashinsky equation (7.33), and we take the exact solution $u(x, t)$ as

$$
u(x, t)=e^{-t} x^{4}(1-x)^{4}, \quad 0<x<1, \quad t>0,
$$

with a suitable $f(x, t)$. The discrete solution $\mathfrak{v}$ is again obtained by the scheme (7.35). In Table 6 we display numerical results. We have picked $\Delta t=h^{2}$, where $h=1 / N$, and the final time is $t=0.25$. Observe in the right panel that fourth-order accuracy is achieved for $u$ and $\frac{\partial u}{\partial x}$. In Figure 11 we display the exact solution $u(x, t)$ at (solid line) $t=0.25$ and the computed solution $\mathfrak{v}$ (circles)for $N=32$ (left) and $N=64$ (center) 

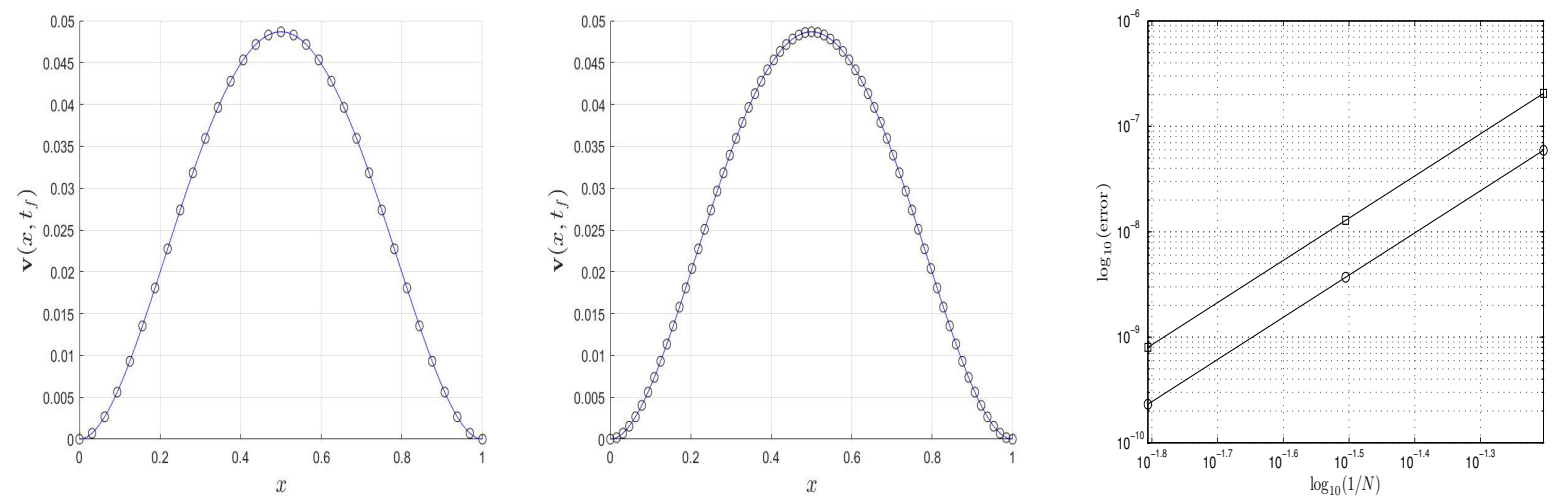

Figure 10. First KS numerical example (7.33): Exact solution (solid line) and computed solution (circles) for $N=32$ (left), $N=64$ (center). The convergence rate is displayed in the right panel for $u$ (circles) and $\frac{\partial u}{\partial x}$ (squares).

at $t=0.25$. Notice that even with a coarse mesh $N=16$ the match is excellent. The right panel shows the fourth order convergence rate for $u$ and $u_{x}$.
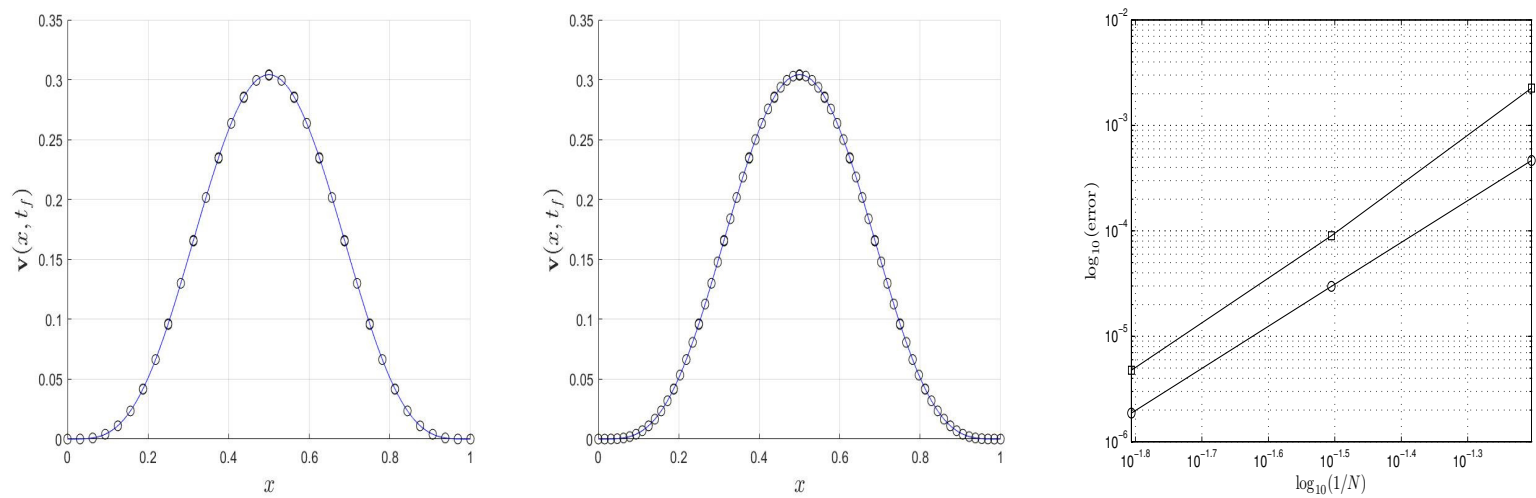

Figure 11. Second KS numerical example: Exact solution (solid line) and computed solution (circles) for $N=32$ (left) and $N=64$ (center) The convergence rate for the KS equation is documented in the right panel for $u$ (circles) and $u_{x}$ (squares).

(3) Third numerical example. We consider again the Kuramoto-Sivashinsky equation (7.33). This time we take the exact solution $u(x, t)[31]$ as

$$
u(x, t)=c+(15 / 19) \sqrt{11 / 19}\left(-9 \tanh \left(k\left(x-c t-x_{0}\right)\right)+11 \tanh ^{3}\left(k\left(x-c t-x_{0}\right)\right), \quad x \in[-30,30],\right.
$$

with no forcing term (i.e. $f(x, t)=0$ ). Here $c=-0.1, k=0.5 \sqrt{11 / 19}$ and $x_{0}=-10$. The discrete solution $\mathfrak{v}$ is again obtained by the scheme (7.35). In Table 7 we display numerical results for (7.35). for the fourth-order scheme. We have picked $\Delta t=h^{2}$, where $h=1 / N$, and the final time is $t=1$. Observe in the right panel that fourth-order accuracy is achieved for $u$ and $\frac{\partial u}{\partial x}$. In Figure 12 we display the exact solution $u(x, t)$ at (solid line) $t=1$ and the computed solution $\mathfrak{v}$ (circles) for $N=121$ (left) and $N=961$ (center) at $t=1$. Notice that even with a coarse mesh $N=121$ the match is very good. The right panel shows the fourth order convergence rate for $u$ and $u_{x}$. 

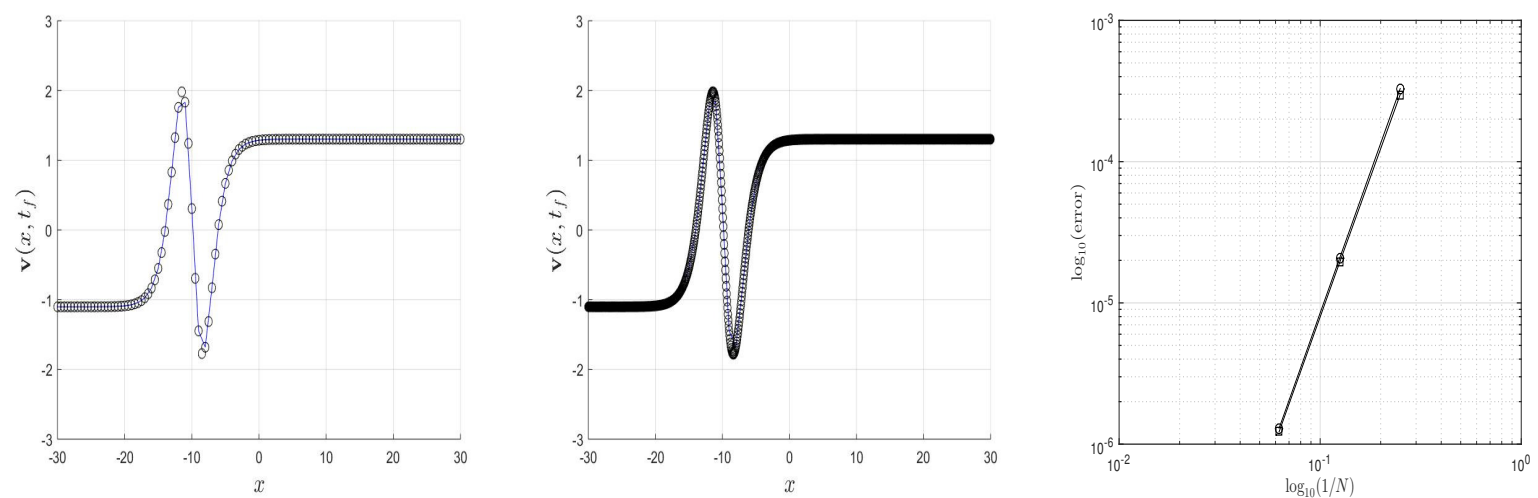

Figure 12. Third KS numerical example: Exact solution (solid line) and computed solution (circles) for $N=121$ (left) and $N=961$ (center) The convergence rate for the KS equation is documented in the right panel for $u$ (circles) and $\frac{\partial u}{\partial x}$ (squares).

\section{ApPEndix A. TAYlor expansions}

In this appendix we collect Taylor expansions of the finite difference operators introduced in the text. For $u(x)$ a regular function, we call $u^{*}$ the restriction of $u(x)$ to the grid. For the Hermitian derivative $\left(u^{*}\right)_{x, j}$ we have ${ }^{2}$

$$
\left(u_{x}^{*}\right)_{j}=\left(\partial_{x} u\right)_{j}^{*}-\frac{h^{4}}{180}\left(\partial_{x}^{5} u\right)_{j}^{*}+O\left(h^{6}\right),
$$

and for the operator $\delta_{x}\left(u^{*}\right)_{x}$ :

$$
\delta_{x}\left(u_{x}^{*}\right)_{j}=\left(\partial_{x}^{2} u\right)_{j}^{*}+\frac{h^{2}}{6}\left(\partial_{x}^{4} u\right)_{j}^{*}+\frac{h^{4}}{360}\left(\partial_{x}^{6} u\right)_{j}^{*}-\frac{h^{6}}{15120}\left(\partial_{x}^{8} u\right)_{j}^{*}+O\left(h^{8}\right) .
$$

On the other hand the centered operator $\delta_{x}^{2} u_{j}^{*}$ has the expansion

$$
\delta_{x}^{2} u_{j}^{*}=\left(\partial_{x}^{2} u\right)_{j}^{*}+\frac{h^{2}}{12}\left(\partial_{x}^{4} u\right)_{j}^{*}+\frac{h^{4}}{360}\left(\partial_{x}^{6} u\right)_{j}^{*}+\frac{h^{6}}{20160}\left(\partial_{x}^{8} u\right)_{j}^{*}+O\left(h^{8}\right) .
$$

Since the coefficient in factor of $h^{4}$ is $1 / 360$ in (A.2) and (A.3) there is a cancellation when evaluating the DBO operator $\delta_{x}^{4} u_{j}^{*}=12\left(\delta_{x} u_{x, j}^{*}-\delta_{x}^{2} u_{j}^{*}\right) / h^{2}$. This gives

$$
\delta_{x}^{4} u_{j}^{*}=\left(\partial_{x}^{4} u\right)_{j}^{*}-\frac{h^{4}}{720}\left(\partial_{x}^{8} u\right)_{j}^{*}+O\left(h^{6}\right) .
$$

Finally the fourth order accuracy of the modified operator $\tilde{\delta}_{x}^{2}$ in (3.10) is seen in the expansion

$$
\tilde{\delta}_{x}^{2} u_{j}^{*}=\left(\partial_{x}^{2} u\right)_{j}^{*}+\frac{h^{4}}{360}\left(\partial_{x}^{6} u\right)_{j}^{*}+O\left(h^{8}\right) .
$$

The small constants in the truncation errors observed in (A.1), (A.4) and (A.5) partly explain the accuracy observed in the numerical results.

\section{REFERENCES}

[1] S. Agmon, Lectures on Elliptic Boundary Value Problems, AMS Chelsea Publishing , 2010.

[2] J. Becker, G. Grün, M. Lenz and M. Rumpf, Numerical methods for fourth-order nonlinear degenerate diffusion problems, Applications of Math. 47 (2002), 517-543.

[3] M. Ben-Artzi, J-P. Croisille, D. Fishelov and S. Trachtenberg, A Pure-Compact Scheme for the streamfunction formulation of Navier-Stokes equations, J. Comput. Phys, 205, (2005), 640-664.

[4] M. Ben-Artzi, J.-P. Croisille and D. Fishelov, Convergence of a compact scheme for the pure streamfunction formulation of the unsteady Navier-Stokes system, SIAM J. Numer. Anal. 44 (2006), 1997-2024.

[5] M. Ben-Artzi, J-P. Croisille and D. Fishelov, A High Order Compact Scheme for the Pure-Streamfunction Formulation of the Navier-Stokes Equations, J. Sci. Comput., 42, (2010), 216-250.

[6] M. Ben-Artzi, J.-P. Croisille and D. Fishelov, "Navier-Stokes Equations in Planar Domains", Imperial College Press, 2013.

\footnotetext{
${ }^{2}$ The Taylor expansions are obtained using a symbolic package
} 
[7] M. Ben-Artzi, J-P. Croisille and D. Fishelov, A fast direct solver for the biharmonic problem in rectangular grid, SIAM J. Scientific Comput., 31, (2008), 303-333.

[8] M. Ben-Artzi, J.-P. Croisille, D. Fishelov and R. Katzir, Discrete fourth-order Sturm-Liouville problems, IMA J. Numer. Anal., $38,3,(2018), 1485-1522$.

[9] A. L. Bertozzi, N. Ju and H.-W. Lu, A biharmonic-modified forward time stepping method for fourth order nonlinear diffusion equations, Disc. Cont. Dynamical Sys., 29, (2011), 1367-1391.

[10] P. Bjørstad, Fast numerical solution of the biharmonic Dirichlet problem on rectangles, SIAM J. Numer. Anal., 20, (1983), 59-71.

[11] S. Boscarini, F. Filbet and G. Russo, High order semi-implicit schemes for time dependent partial differential equations , J. Sci. Comput. 68 (2016), 975-1001.

[12] J.C. Butcher, Numerical methods for ordinary differential equations, 2cd ed. Wiley, 2008.

[13] Y. Cheng and C.-W. Shu, A discontinuous Galerkin finite element method for time dependent partial differential equations with higher order derivatives, Math. Comp. 77 (2008), 699-730.

[14] P. Danumjayau, Finite element methods for one dimensional fourth order semilinear differential equation, Int. J. Appl. Comput. Math. 2 (2016), 395-410.

[15] E.B. Davies, "Spectral theory of differential operators", Cambridge studies in advanced mathematics, 1995.

[16] D. Fishelov, Semi-discrete time-dependent fourth-order problems on an interval: error estimate, in "Numerical Mathematics and Advanced Applications - ENUMATH 2013", Eds. Assyr Abdulle, Simone Deparis, Daniel Kressner, Fabio Nobile, and Marco Picasso, Lecture Notes in Computational Science and Engineering, 103, 133-142, Springer-Verlag, 2015.

[17] D. Fishelov, M. Ben-Artzi and J.-P. Croisille, Recent advances in the study of a fourth-order compact scheme for the onedimensional biharmonic equation, J. Sci. Comput., 53 (2012), 55-79.

[18] D. Fishelov, M. Ben-Artzi and J.-P. Croisille, Fourth-order convergence of a compact scheme for the one-dimensional biharmonic equation, AIP Conf. Proceedings, 1479, (2012), 1101-1104.

[19] D. Fishelov and N. Sochen, Image inpainting via fluid equations, Information Technology: Research and Education, ITRE'06 (2006), 23-25.

[20] J. B. Greer and A. L. Bertozzi, $H_{1}$ solutions of a class of fourth order nonlinear equations for image processing, Discerete and Continuous Dynamical systems, 10 (2004), 349-366.

[21] J. A. Gregory, D. Fishelov, B. Schiff, and J. R. Whiteman, Local Mesh Refinement with Finite Elements for Elliptic Problem, J. Comput. Phys., Discerete and Continuous Dynamical systems, 29 (1978), 133-140.

[22] A. Iserles, "A First Course in the Numerical Analysis of Differential Equations", Cambridge University Press (2008).

[23] A.-K. Kassam and L. N. Trefethen, Fourth-order time-stepping for stiff PDEs, SIAM J. Sci. Comput. 26 (2005), $1214-1233$.

[24] R. Kupferman, A central-difference scheme for a pure stream function formulation of incompressible viscous flow, SIAM J. Sci. comput., 23, (2001), 1-18.

[25] Y. Kuramoto and T. Tsuzuki, Persistent propagation of concentration waves in dissipative media far from thermal equilibrium, Progr. Theoret. Phys.,55, (1976), 356-369.

[26] M. Lysaker, T. Tsuzuki and X.-C. Tai, Noise removal using fourth-order partial differential equation with applications to medical magnetic resonance images in space and time, IEEE Transactions on Image processing, 12 (2003), 1597-1590.

[27] R.D. Russell , J.F. Williams and X.Xu, MOVCOL4: A moving mesh code for fourth-order time-dependent partial differential equations, SIAM J. Sci. Comput., 29, (2007), 197-220.

[28] G. Sivashinsky, Nonlinear analysis of hydrodynamic instability in laminar flames I. Derivation of basic equations, Acta Astron. 4 (1977), 1177-1206.

[29] G. Sivashinsky and D. Michelson, On irregular wavy flow of a liquid film down a vertical plane, Progr. Theoret. Phys., 63 (1980), $2112-2114$.

[30] E. Süli. I.Mozolevski, hp-version interior penalty DGFEMs for the biharmonic equation, Comput. Methods Appl. Mech. Eng., 196, (2007), 1851-1863.

[31] Y. Xu and C.-W. Shu, Local discontinuous Galerkin methods for the Kuramoto-Sivashinsky equations and the Ito-type coupled KdV equations, Comput. Methods Appl. Mech. Eng.,195,(2006), 3430-3447.

[32] J. Yan and C.-W. Shu, Local discontinuous Galerkin methods for partial differential equations with higher order derivatives, J. Sci. Comput.,17, (2002), 27-47. 


\section{ApPendix B. TABles}

\begin{tabular}{||l|l|l|l|l|l|l|l||}
\hline \hline mesh & $N=10$ & Rate & $N=20$ & Rate & $N=40$ & Rate & $N=80$ \\
\hline$|\mathfrak{e}|_{\infty}$ & $6.65(-5)$ & 4.07 & $3.95(-6)$ & 4.02 & $2.43(-7)$ & 4.00 & $1.52(-8)$ \\
\hline$|\mathfrak{e}|_{\infty, \text { DG }}[32]$ & $6.10(-5)$ & 4.69 & $2.35(-6)$ & 4.06 & $1.40(-7)$ & 4.00 & $8.75(-9)$ \\
\hline \hline
\end{tabular}

TABLE 1. Biharmonic periodic problem (7.6) with exact solution $u(x, t)=\sin (x) \exp (-t)$ on $x \in[0,2 \pi)$ at final time is $t_{f}=\ln (2)$. The first line indicates the errors obtained by the scheme (7.7). The second line indicates the errors obtained by a piecewise cubic discontinuous Galerkin method [32] (without postprocessing). The time scheme Radau-IIA is used for (7.7). The time step is $\Delta t=K h^{4 / 3}$ with 5 iterations for $N=10$. There are 80 iterations for the grid $N=80$. Note that the DG scheme has 4 times more unknowns ( 4 unknowns per cell) that the compact scheme (one unknown per point).

\begin{tabular}{||l|l|l|l|l|l|l|l||}
\hline \hline mesh & $N=8$ & Rate & $N=16$ & Rate & $N=32$ & Rate & $N=64$ \\
\hline$|\mathfrak{e}|_{\infty}$ & $2.46(+0)$ & 4.02 & $1.64(-1)$ & 4.13 & $1.01(-2)$ & 3.99 & $5.83(-4)$ \\
\hline \hline
\end{tabular}

TABLE 2. Equation(7.8) with $a=1, b=-1$ discretized by (7.9). The exact solution is $u(x, t)=$ $x^{4}(1-x)^{4} e^{t}$. The initial time $t_{0}=0$ and the final time is $t_{f}=10$. The time scheme is the Radau-IIA scheme. The time step is $\Delta t=C h^{4 / 3}$ (8 time iterations for the coarsest grid $N=8$ ). There is 125 iterations on the finest grid $N=64$.

\begin{tabular}{||l|l|l|l|l|l|l|l||}
\hline \hline mesh & $N=32$ & Rate & $N=64$ & Rate & $N=128$ & Rate & $N=256$ \\
\hline$|\mathfrak{e}|_{\infty}$ & $1.23(-1)$ & 6.70 & $1.18(-3)$ & 4.28 & $6.10(-7)$ & 4.07 & $3.65(-8)$ \\
\hline \hline
\end{tabular}

TABLE 3. Equation (7.8) with $a=1, b=-1$ with exact solution: $u(x, t)=p(x) \sin \left(1 / q_{\varepsilon}(x)\right) \sin (2 \pi t)$, $\varepsilon=0.05$. The initial time is $t_{0}=0$ and the final time is $t_{f}=0.75$. The time scheme is the Radau-IIA scheme. The time step is $\Delta t=K h^{4 / 3}$ with 10 iterations for $N=32$. There is 160 iterations on the finest grid $N=256$.

\begin{tabular}{||l|l|l|l|l|l|l|l||}
\hline \hline mesh & $N=32$ & Rate & $N=64$ & Rate & $N=128$ & Rate & $N=256$ \\
\hline$|\mathfrak{e}|_{\infty}$ & $1.23(-1)$ & 6.73 & $1.20(-3)$ & 4.33 & $7.50(-5)$ & 4.08 & $7.63(-6)$ \\
\hline \hline
\end{tabular}

TABLE 4. Equation (7.31) with exact solution: $u_{\varepsilon}(x, t)=p(x) \sin \left(1 / q_{\varepsilon}(x)\right) \sin (2 \pi t)$, with the particular value $\varepsilon=0.05$. Initial time $t_{0}=0$, Final time $t_{f}=0.75$. The time scheme is the Radau-IIA scheme. The time step is $\Delta t=C h^{4 / 3}$ (10 time iterations for $\left.N=32\right)$.

\begin{tabular}{||c|c|c|c|c|c||}
\hline \hline Mesh & $N=16$ & Rate & $N=32$ & Rate & $N=64$ \\
\hline$|\mathfrak{e}|_{h}$ & $5.9269(-8)$ & 4.00 & $3.7045(-9)$ & 4.00 & $2.3129(-10)$ \\
\hline$\left|\mathfrak{e}_{x}\right|_{h}$ & $2.0558(-7)$ & 4.00 & $1.2840(-8)$ & 4.00 & $8.0158(-10)$ \\
\hline \hline
\end{tabular}

TABLE 5. Compact scheme for KS equation (7.33) with exact solution $u=e^{-t} x^{2}(x-1)^{2}$ on $[0,1]$. We display $|\mathfrak{e}|_{h}$ and $\left|\mathfrak{e}_{x}\right|_{h}$ the errors in $u$, and $u_{x}$, respectively at $\mathrm{t}=0.25$. The time step is $\Delta t=h^{2}$. 


\begin{tabular}{||c|c|c|c|c|c||}
\hline \hline Mesh & $N=16$ & Rate & $N=32$ & Rate & $N=64$ \\
\hline$|\mathfrak{e}|_{h}$ & $4.6595(-4)$ & 3.97 & $2.9750(-5)$ & 3.99 & $1.8702(-6)$ \\
\hline$\left|\mathfrak{e}_{x}\right|_{h}$ & $2.2606(-3)$ & 4.65 & $8.9829(-5)$ & 4.24 & $4.7682(-6)$ \\
\hline \hline
\end{tabular}

TABLE 6. Compact scheme for KS equation (7.33) with exact solution $u=e^{-t} x^{4}(x-1)^{4}$ on $[0,1]$. We display $|\mathfrak{e}|_{h}$ and $\left|\mathfrak{e}_{x}\right|_{h}$, the errors in $u$, and $u_{x}$, respectively at $t=0.25$. The time step is $\Delta t=h^{2}$.

\begin{tabular}{||c|c|c|c|c|c||}
\hline \hline Mesh & $N=241$ & Rate & $N=481$ & Rate & $N=961$ \\
\hline$|\mathfrak{e}|_{h}$ & $3.2873(-4)$ & 3.99 & $2.0752(-5)$ & 4.00 & $1.2984(-6)$ \\
\hline$\left|\mathfrak{e}_{x}\right|_{h}$ & $2.9822(-4)$ & 3.95 & $1.9332(-5)$ & 3.98 & $1.2246(-6)$ \\
\hline \hline
\end{tabular}

TABLE 7. Compact scheme for KS equation (7.33) with exact solution $u=u(x, t)=c+$ $(15 / 19) \sqrt{(11 / 19)}\left(-9 \tanh \left(k\left(x-c t-x_{0}\right)\right)+11 \tanh ^{3}\left(k\left(x-c t-x_{0}\right)\right)\right.$. We display $|\mathfrak{e}|_{h}$ and $\left|\mathfrak{e}_{x}\right|_{h}$, the errors in $u$, and $u_{x}$, respectively at $t=1$. The time step is $\Delta t=h^{2}$.

Matania Ben-Artzi: Institute of Mathematics, The Hebrew University, Jerusalem 91904, Israel

E-mail address: mbartzi@math.huji.ac.il

Jean-Pierre Croisille: Département de Mathématiques, Univ. de Lorraine, Metz 57045, France and CNRS, Institut Élie Cartan de Lorraine,UMR 7502, Metz, F-57045, France

E-mail address: jean-pierre.croisille@univ-lorraine.fr

Dalia Fishelov:Department of Mathematics, Afeka - Tel-Aviv Academic College of Engineering, 38 Mivza Kadesh St., Tel-Aviv 69107, IsRAel

E-mail address: daliaf@afeka.ac.il 